\title{
Deep Bore Hole Instrumentation Along San Francisco Bay Bridges
}

Lawrence Hutchings, Paul Kasameyer, William Foxall, Jennifer Hollfelder and Christine Turpin;

Lawrence Livermore National Laboratory

Tom McEvilly and Richard Clymer; University of California, Berkeley

Pat Hipley, Lalliana Mualchin, John Bowman, and Mark Palmer; Caltrans, Sacramento, CA

Steven Jarpe, Institute of Crustal Studies; Santa Barbara, CA

William Bakun; United States Geological Survey (USGS)

October 1998

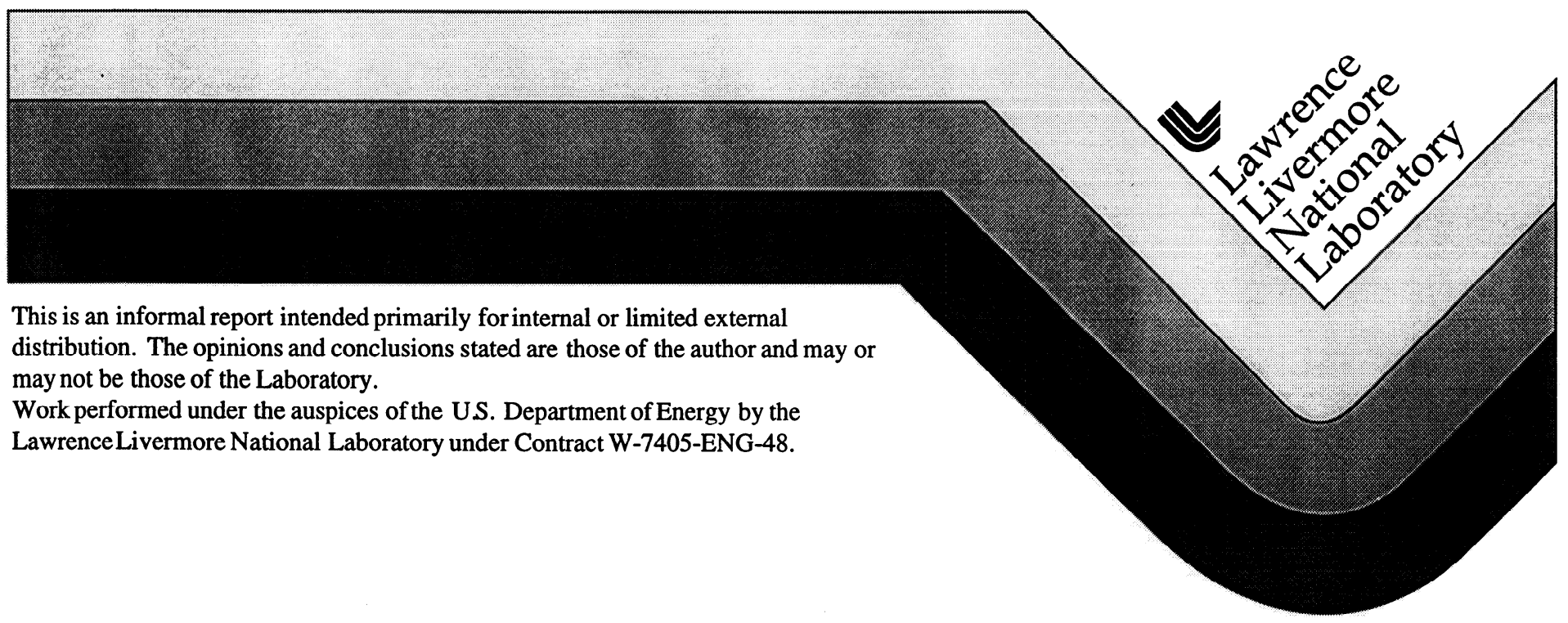




\section{DISCLAIMER}

This document was prepared as an account of work sponsored by an agency of the United States Government. Neither the United States Government nor the University of California nor any of their employees, makes any warranty, express or implied, or assumes any legal liability or responsibility for the accuracy, completeness, or usefulness of any information, apparatus, product, or process disclosed, or represents that its use would not infringe privately owned rights. Reference herein to any specific commercial product, process, or service by trade name, trademark, manufacturer, or otherwise, does not necessarily constitute or imply its endorsement, recommendation, or favoring by the United States Government or the University of California. The views and opinions of authors expressed herein do not necessarily state or reflect those of the United States Government or the University of California, and shall not be used for advertising or product endorsement purposes.

This report has been reproduced directly from the best available copy.

Available to DOE and DOE contractors from the Office of Scientific and Technical Information

P.O. Box 62, Oak Ridge, TN 37831

Prices available from (615) 576-8401, FTS 626-8401

Available to the public from the

National Technical Information Service

U.S. Department of Commerce

5285 Port Royal Rd.,

Springfield, VA 22161 


\title{
Deep Bore Hole Instrumentation Along San Francisco Bay Bridges
}

Lawrence Hutchings, Paul Kasameyer, William Foxall, Jennifer Hollfelder, and Christine Turpin; Lawrence Livermore National Laboratory Tom McEvilly and Richard Clymer, University of California, Berkeley Pat Hipley, Lalliana Mualchin, John Bowman, and Mark Palmer, Caltrans, Sacramento, California Steve Jarpe, Institute of Crustal Studies, Santa Barbara, California William Bakun, USGS environmental

\begin{abstract}
The Bay Bridges down hole network consists of sensors in bore holes that are drilled 100' into bedrock around and in the San Francisco Bay. Between 2 and 8 instruments have been spaced along the Dumbarton, San Mateo, Bay, and San Rafael bridges. The instruments will provide multiple use data that is important to geotechnical, structural engineering, and seismological studies. The holes are between 100 and $1000 \mathrm{ft}$ deep and were drilled by Caltrans. There are twentyone sensor packages at fifteen sites. Extensive financial support is being contributed by Caltrans, UCB, LBL, LLNL-LDRD, U.C. Campus/Laboratory Collaboration (CLC) program, and USGS. The down hole instrument package contains a three component HS-1 seismometer and three orthogonal Wilcox 731 accelerometers, and is capable of recording a micro $\mathrm{g}$ from local $\mathrm{M}=1.0$ earthquakes to $0.5 \mathrm{~g}$ strong ground motion form large Bay Area earthquakes.
\end{abstract}

\section{Introduction}

The Bay Bridges down hole network consists of recordings in bore holes that are drilled $100 \mathrm{ft}$ into bedrock along and in the San Francisco Bay (Figure 1). Between 1 and 8 instruments have been spaced along the Dumbarton, San Mateo, Bay, Carquinez, and San Rafael bridges. Tables 1 - 5 list recording site information, and Figures 2 - 6 show profile locations of the instrument locations. In addition, two vertical arrays exist at the Dumbarton bridge with additional sensors at the surface and at $200 \mathrm{ft}$ (Table 1). Two sensors are currently located at the surface at the Bay Bridge and are waiting drill holes. Prior to this study few seismic recording instruments existed in bedrock in San Francisco Bay. This left a recording gap for engineering studies of the Bay bridges and in seismicity studies of the Bay Area. Figures 7 and 8 show earthquakes recorded by instruments along the Bay and Dumbarton bridges. The Bridges network is part of a larger Hayward Fault Digital Network, see Figure 9.

There are six primary areas of research by LLNL that will be enhanced by the bore hole instrumentation: 1) developing realistic predictions of strong ground motion at multiple input points along long span bridges, 2) examining ground motion variability in bedrock, 3) calibrating soil response models, 4) developing bridge response calculations with multiple support input motions, 5) evaluate the seismicity of potentially active faults in the San Francisco Bay, and 6) record strong ground motion.

Key to these studies is LLNL's effort to exploit the information available in weak ground motions (generally from earthquakes $<M=3.0$ ) to enhance predictions of seismic hazards. Although strong 
ground motion recordings are essential to calibrate models and understand the hazard of future earthquakes, we can obtain weak ground motion data immediately, whereas it may be years before strong motion data is recorded. Following is an expansion of research goals utilizing recordings from the Bridges Network.

1) prediction of strong ground motion: LLNL is developing a methodology of using weak ground motion to synthesize linear response strong ground motion and incorporating this with constraints on fault rupture scenarios to predict strong ground motion. These computations provide estimates of the full wavetrain ground motion at multiple points along long span structures.

2) ground motion variability: Recent studies have demonstrated the high variability of strong ground motion with site conditions. Recordings along Bay bridges will be used both to improve calculations of ground motions for bridges, and to research the spatial sensitivity and significance of site variability to structures.

3) soils response: LLNL is researching means of using weak ground motion to constrain soils models for non-linear computations. Current research has shown that low strain constitutive properties are significant to non-linear ground motion computations, and that these values can be significantly improved by an iterative process of matching weak motion solutions.

4) bridge response calculations: Current developments in structural dynamics allow non-linear, three-dimensional calculation of bridge response. This requires realistic full wavetrain input ground motions. LLNL is conducting research on the sensitivity of synthetic ground motions to accurate non-linear computations, and the significance of utilizing multiple support input calculations.

5) seismicity: Location of small earthquakes within the Bay that may indicate the existence of active faults will be made possible with the instrumentation. Very small earthquakes $(\mathrm{M}<2)$ cannot be recorded adequately to determine accurate locations by regional networks.

6) strong ground motion: Strong ground motion from previous earthquakes gives a good indication of what might be expected from future earthquakes. In addition recent earthquakes have demonstrated the high variability of strong ground motion so that an array of strong ground motion recordings will give a better understanding of the ground motion variability from future earthquakes.

\section{Instrumentation}

The down-hole sensor package is manufactured at LBL under the direction to Dr. Tom McEvilly, and is the same package used by the USGS and LBL for the Hayward Fault Digital Recording Network. This package contains three orthogonal Oyo HS-1 4.5 Hz geophones and a three orthogonal Wilcoxon 731s 10v/g accelerometers. The dynamic range of the Wilcoxon package is from a micro-g to $0.5 \mathrm{~g}$ acceleration, and is flat to frequency response from 0.1 to $300 \mathrm{~Hz}$. This allows recording of $\mathrm{M}=1.0$ to $0.5 \mathrm{~g}$ strong ground motion form large Bay Area earthquakes. Typically, the Wilcoxon's are recorded over two dynamic ranges to capture weak and strong ground 
motions, and HS-1's are used as a backup for weak ground motion recording. A Portable Data Acquisition System (DAS) is used to record the data. The DAS are 16 bit Refraction Technology 72A series seismographs is used for most sites. Three site utilize Quantera-4120 recorders. The signals are sampled at $200 \mathrm{sps}$. The data is processed and managed at UC Berkeley.

\section{Geology of San Francisco Bay}

San Francisco Bay is composed of two distinct units; bedrock and a younger unconsolidated sediment sequence which can be further subdivided into the Alameda formation (oldest), San Antonio formation, Posey formation, Merritt formation and the Bay Mud (youngest). Figure 10 shows the subsurface geology beneath the San Francisco- Oakland Bay Bridge. (Trask, P. and Rolston, R., 1951, Engineering Geology of San Francsisco Bay, California: Bull. Geol. Soc. Am.: v. 62, pp. 10791110.)

\begin{tabular}{|l|l|}
\hline \multicolumn{1}{|c|}{ Unit } & \multicolumn{1}{c|}{ Geologic Characteristics } \\
\hline Bedrock & $\begin{array}{l}\text { Composed of the Franciscan formation (Mesozoic). The Franciscan } \\
\text { formation contains interbedded feldspathic sandstone, graywacke, } \\
\text { siltstone, shale, limestone, radiolarian chert, metavolcanic rocks, } \\
\text { and glaucophane schists. The total thickness of this unit is unknown } \\
\text { but has been estimated to be at least 10,000 feet thick and at most } \\
50,000 \text { feet thick. (Figure 11). }\end{array}$ \\
\hline Alameda formation & $\begin{array}{l}\text { Composed of layers of firm sand, silt, clay, and fine gravel. The for- } \\
\text { mation commonly appears gray but can be greenish gray or brown- } \\
\text { ish gray. The gravel contains well rounded pebbles (up to lin in } \\
\text { diameter) from the Franciscan formation. Plant fragments can be } \\
\text { found throughout the unit but seem to be heavily concentrated } \\
\text { within the upper portion. On the west side of the San Francisco Bay, } \\
\text { at a depth of } 280 \text { feet, a layer of clean white volcanic ash is inter- } \\
\text { bedded within the unit. The volcanic ash is a dacitic vitric tuff } \\
\text { which contains 10\% crystals (feldspar, hornblende, quartz) and } \\
\text { 90\% glass. }\end{array}$ \\
\hline San Antonio formation & $\begin{array}{l}\text { The unit can be divided into three distinct layers. The first layer } \\
\text { consists of a firm silty clay. The second layer contains fine- to } \\
\text { medium-grained sand and silty clay with shell fragments. The sec- } \\
\text { ond layer is not found on the western side of the San Francisco Bay. } \\
\text { The third layer is composed of gray to greenish gray fine grained } \\
\text { clay with interbedded layers of sand or sandy gravel containing } \\
\text { Franciscan pebbles. This layer contains a continuous bed of plant } \\
\text { fragments near the base. }\end{array}$ \\
\hline
\end{tabular}

Acknowledgement: Work performed under the auspices of the U.S. Department of Energy by Lawrence Livermore National Laboratory under Contract W-7405-ENG-48. 
Table 2: Bay Bridge Recording sites

\begin{tabular}{|l|c|c|c|c|c|l|}
\hline \multicolumn{1}{|c|}{ i.d. } & sensors & latitude & longitude & $\begin{array}{c}\text { depth } \\
(\mathrm{m})\end{array}$ & $\begin{array}{c}\text { sensor h1, } \\
\text { h2 +090 }\end{array}$ & Recording \\
\hline \hline E07 & Wil-731A,HS-1 & 37.81847 & 122.34688 & ---- & ------ & 2/96-present \\
\hline E17 & Wil-731A,HS-1 & 37.82086 & 122.33534 & 159.7 & ----- & $8 / 95$-present \\
\hline $\begin{array}{l}\text { E23 } \\
\text { (BBEB) }\end{array}$ & HS-1 & 37.82167 & 122.32867 & 150 & ----- & $3 / 94-10 / 95$ \\
\hline
\end{tabular}

Table 3: San Rafael Bridge Recording sites

\begin{tabular}{|l|c|c|c|c|c|c|}
\hline i.d. & sensors & latitude & longitude & depth & $\begin{array}{c}\text { sensor } \mathrm{h} 1, \\
\mathrm{~h} 2+090\end{array}$ & Recording \\
\hline \hline P58 & Wil-731A,HS-1 & 37.93372 & 122.41313 & $---\mathrm{m}$ & $\mathrm{N} 0^{\circ} \mathrm{E}$ & 6/97-present \\
\hline P34 & Wil-731A,HS-1 & 37.9358 & 122.4454 & ----- & & 8/97-present \\
\hline
\end{tabular}

Table 4: San Mateo Bridge Recording sites

\begin{tabular}{|c|c|c|c|c|c|c|}
\hline i.d. & sensors & latitude & longitude & depth & $\begin{array}{c}\text { sensor } \mathrm{h} 1, \\
\mathrm{~h} 2+090\end{array}$ & Recording \\
\hline \hline P343 & Wil-731A,HS-1 & 37.786 & 122.391 & $298.4 \mathrm{~m}$ & $\mathrm{~N} 0^{\circ} \mathrm{E}$ & not recorded \\
\hline
\end{tabular}

Table 5: Carqueniz Bridge Recording sites

\begin{tabular}{|c|c|c|c|c|c|c|}
\hline i.d. & sensors & latitude & longitude & depth & $\begin{array}{c}\text { sensor } \mathrm{h} 1, \\
\mathrm{~h} 2+090\end{array}$ & Recording \\
\hline \hline CRSB & Wil-731A,HS-1 & 37.786 & 122.391 & $38.4 \mathrm{~m}$ & $\mathrm{~N} 0^{\circ} \mathrm{E}$ & 6/98-present \\
\hline
\end{tabular}


Table 6: Events Recorded in the Study

\begin{tabular}{|l|l|l|l|l|l|}
\hline \multicolumn{1}{|c|}{ Earthquake } & \multicolumn{1}{|c|}{ Time } & \multicolumn{1}{|c|}{ Latitude } & \multicolumn{1}{c|}{ Longitude } & \multicolumn{1}{c|}{ Depth } & Magnitude \\
\hline $1996 / 02 / 04$ & $23: 27: 28.74$ & 37.8798 & -122.3200 & 10.89 & 1.60 \\
\hline $1996 / 03 / 20$ & $00: 31: 27.07$ & 37.7768 & -122.1915 & 11.03 & 2.00 \\
\hline $1996 / 04 / 12$ & $08: 52: 28.73$ & 38.1750 & -122.4807 & 12.34 & 2.40 \\
\hline $1996 / 04 / 14$ & $13: 36: 21.37$ & 37.7743 & -122.3703 & 14.51 & 1.60 \\
\hline $1996 / 04 / 24$ & $05: 21: 05.06$ & 37.7657 & -122.1458 & 11.16 & 2.20 \\
\hline $1996 / 05 / 14$ & $17: 51: 08.00$ & 37.9118 & -122.2825 & 6.43 & 2.10 \\
\hline $1996 / 05 / 15$ & $04: 11: 36.96$ & 37.8017 & -122.1988 & 10.50 & 1.90 \\
\hline $1996 / 05 / 21$ & $20: 50: 20.16$ & 37.3593 & -121.7227 & 8.14 & 4.50 \\
\hline $1996 / 05 / 30$ & $02: 50: 45.66$ & 37.8712 & -122.2387 & 11.16 & 2.00 \\
\hline $1996 / 05 / 31$ & $08: 36: 47.55$ & 37.8745 & -122.2415 & 9.51 & 1.80 \\
\hline $1996 / 07 / 01$ & $04: 33: 05.12$ & 37.7148 & -122.5447 & 10.94 & 3.10 \\
\hline $1996 / 07 / 04$ & $19: 53: 00.53$ & 38.3755 & -122.6115 & 8.58 & 2.70 \\
\hline $1996 / 08 / 25$ & $23: 20: 48.27$ & 37.8513 & -122.2128 & 9.33 & 2.00 \\
\hline $1996 / 10 / 31$ & $23: 09: 43.37$ & 37.7553 & -122.1667 & 9.46 & 2.30 \\
\hline $1996 / 12 / 24$ & $16: 16: 54.79$ & 37.7355 & -122.5455 & 6.56 & 2.20 \\
\hline $1996 / 12 / 31$ & $05: 39: 41.80$ & 37.8787 & -122.2495 & 9.85 & 1.50 \\
\hline $1997 / 02 / 05$ & $00: 25: 41.05$ & 38.3647 & -122.6513 & 6.75 & 3.70 \\
\hline $1997 / 02 / 14$ & $10: 35: 31.48$ & 37.7072 & -122.1168 & 7.85 & 1.60 \\
\hline $1997 / 02 / 17$ & $16: 51: 14.89$ & 38.7943 & -122.7450 & 1.04 & 1.10 \\
\hline $1997 / 02 / 20$ & $13: 43: 38.60$ & 37.8415 & -122.2280 & 5.61 & 1.80 \\
\hline $1997 / 03 / 11$ & $06: 30: 16.33$ & 37.7123 & -122.5663 & 5.22 & 3.50 \\
\hline $1997 / 03 / 11$ & $06: 32: 36.58$ & 37.7137 & -122.5653 & 4.96 & 2.40 \\
\hline $1997 / 03 / 11$ & $06: 33: 53.43$ & 37.7137 & -122.5663 & 4.92 & 3.40 \\
\hline $1997 / 03 / 11$ & $06: 41: 33.68$ & 37.7125 & -122.5658 & 4.78 & 2.30 \\
\hline $1997 / 03 / 18$ & $13: 50: 05.62$ & 37.4263 & -121.7722 & 8.20 & 2.00 \\
\hline $1997 / 03 / 27$ & $11: 30: 07.00$ & 38.1483 & -121.9372 & 21.55 & 3.40 \\
\hline
\end{tabular}


Table 6: Events Recorded in the Study

\begin{tabular}{|l|l|l|l|l|l|}
\hline \multicolumn{1}{|c|}{ Earthquake } & \multicolumn{1}{|c|}{ Time } & \multicolumn{1}{|c|}{ Latitude } & \multicolumn{1}{c|}{ Longitude } & \multicolumn{1}{c|}{ Depth } & Magnitude \\
\hline $1997 / 03 / 27$ & $13: 38: 08.82$ & 38.1497 & -121.9255 & 21.43 & 3.20 \\
\hline $1997 / 03 / 27$ & $15: 39: 49.01$ & 38.1492 & -121.9365 & 21.65 & 3.70 \\
\hline $1997 / 03 / 27$ & $22: 47: 53.00$ & 38.1500 & -121.9333 & 22.13 & 3.50 \\
\hline $1997 / 03 / 28$ & $04: 26: 31.49$ & 37.3080 & -122.0988 & 5.78 & 2.50 \\
\hline $1997 / 03 / 30$ & $06: 34: 01.38$ & 38.0257 & -122.3765 & 7.32 & 2.30 \\
\hline $1997 / 04 / 01$ & $01: 36: 54.87$ & 38.1492 & -121.9335 & 21.81 & 3.60 \\
\hline $1997 / 04 / 03$ & $17: 31: 22.25$ & 37.8668 & -122.2388 & 10.01 & 1.90 \\
\hline $1997 / 04 / 06$ & $07: 41: 36.05$ & 38.8107 & -122.7743 & 2.76 & 1.20 \\
\hline $1997 / 04 / 14$ & $10: 29: 05.12$ & 38.8430 & -122.7910 & 1.96 & 1.60 \\
\hline $1997 / 04 / 22$ & $11: 18: 08.04$ & 37.4282 & -121.7708 & 10.14 & 3.30 \\
\hline $1997 / 05 / 01$ & $00: 38: 33.72$ & 37.4247 & -121.7695 & 9.02 & 1.90 \\
\hline $1997 / 05 / 01$ & $09: 45: 15.15$ & 37.5083 & -121.6778 & 9.46 & 2.30 \\
\hline $1997 / 05 / 02$ & $09: 02: 20.42$ & 37.5082 & -121.6773 & 9.50 & 2.30 \\
\hline $1997 / 05 / 02$ & $11: 30: 37.62$ & 37.7062 & -122.5175 & 5.20 & 2.50 \\
\hline $1997 / 05 / 02$ & $12: 31: 05.61$ & 37.7070 & -122.5163 & 4.97 & 3.15 \\
\hline $1997 / 05 / 20$ & $09: 22: 32.41$ & 37.5668 & -121.8787 & 9.20 & 1.30 \\
\hline $1997 / 05 / 29$ & $10: 21: 06.83$ & 37.1167 & -121.5210 & 8.06 & 3.59 \\
\hline $1997 / 05 / 29$ & $18: 28: 44.09$ & 37.1190 & -121.5230 & 8.16 & 3.50 \\
\hline $1997 / 06 / 19$ & $00: 35: 29.87$ & 37.7795 & -122.5942 & 3.08 & 2.50 \\
\hline $1997 / 06 / 19$ & $04: 11: 42.66$ & 37.7795 & -122.5963 & 2.96 & 2.20 \\
\hline $1997 / 06 / 22$ & $16: 26: 29.38$ & 37.5782 & -121.9513 & 9.63 & 1.70 \\
\hline $1997 / 06 / 25$ & $01: 11: 34.86$ & 37.8477 & -122.2308 & 4.75 & 1.10 \\
\hline $1997 / 06 / 25$ & $03: 13: 59.46$ & 37.8547 & -122.2253 & 8.09 & 1.60 \\
\hline $1997 / 07 / 04$ & $02: 59: 17.16$ & 37.3053 & -122.3543 & 6.04 & 2.20 \\
\hline $1997 / 07 / 09$ & $16: 14: 47.66$ & 37.9337 & -122.0262 & 11.80 & 2.90 \\
\hline $1997 / 07 / 11$ & $08: 31: 27.02$ & 37.6440 & -122.0452 & 2.08 & 1.60 \\
\hline $1997 / 07 / 14$ & $06: 11: 11.70$ & 37.1722 & -122.3348 & 14.01 & 3.76 \\
\hline
\end{tabular}


Table 6: Events Recorded in the Study

\begin{tabular}{|l|l|l|l|l|l|}
\hline \multicolumn{1}{|c|}{ Earthquake } & \multicolumn{1}{|c|}{ Time } & \multicolumn{1}{|c|}{ Latitude } & \multicolumn{1}{c|}{ Longitude } & \multicolumn{1}{c|}{ Depth } & Magnitude \\
\hline $1997 / 07 / 17$ & $20: 16: 00.69$ & 38.8090 & -122.8147 & 3.87 & 1.30 \\
\hline $1997 / 07 / 30$ & $11: 30: 38.93$ & 37.5715 & -121.6672 & 7.68 & 3.16 \\
\hline $1997 / 08 / 12$ & $22: 01: 59.62$ & 37.5872 & -121.9707 & 6.60 & 2.00 \\
\hline $1997 / 08 / 14$ & $08: 53: 35.67$ & 37.7370 & -122.5477 & 1.84 & 3.02 \\
\hline $1997 / 08 / 17$ & $18: 41: 54.17$ & 37.8682 & -122.2390 & 10.07 & 1.30 \\
\hline $1997 / 08 / 23$ & $14: 26: 18.95$ & 37.3732 & -121.7343 & 6.40 & 2.40 \\
\hline $1997 / 08 / 23$ & $14: 27: 02.59$ & 37.3733 & -121.7340 & 6.34 & 2.00 \\
\hline $1997 / 08 / 27$ & $20: 29: 17.27$ & 38.8448 & -122.7990 & 1.42 & 1.00 \\
\hline $1997 / 08 / 31$ & $00: 24: 13.99$ & 37.7360 & -122.0900 & 9.42 & 2.92 \\
\hline $1997 / 08 / 31$ & $17: 18: 45.07$ & 38.8303 & -122.8630 & 0.80 & 1.10 \\
\hline $1997 / 09 / 03$ & $19: 00: 30.83$ & 38.8007 & -122.7728 & 1.80 & 1.30 \\
\hline $1997 / 09 / 08$ & $00: 10: 38.00$ & 37.8595 & -122.2328 & 9.60 & 1.40 \\
\hline $1997 / 09 / 12$ & $21: 19: 18.84$ & 37.7923 & -122.6233 & 5.20 & 2.00 \\
\hline $1997 / 09 / 18$ & $04: 25: 53.59$ & 37.3708 & -122.1452 & 6.06 & 2.50 \\
\hline $1997 / 09 / 23$ & $09: 52: 20.92$ & 37.1792 & -122.0695 & 16.09 & 2.10 \\
\hline $1997 / 09 / 24$ & $04: 41: 33.71$ & 37.9675 & -122.3390 & 6.98 & 2.50 \\
\hline $1997 / 09 / 26$ & $19: 58: 02.47$ & 37.2863 & -121.6640 & 6.96 & 3.19 \\
\hline $1997 / 10 / 19$ & $05: 23: 54.17$ & 37.4505 & -121.7720 & 7.94 & 1.20 \\
\hline $1997 / 10 / 27$ & $00: 29: 13.68$ & 37.6815 & -121.8393 & 9.65 & 2.50 \\
\hline $1997 / 10 / 27$ & $14: 30: 50.65$ & 37.7265 & -122.5473 & 10.15 & 2.90 \\
\hline $1997 / 11 / 15$ & $15: 51: 59.78$ & 38.8257 & -122.8318 & 1.36 & 1.60 \\
\hline $1997 / 11 / 19$ & $21: 05: 17.94$ & 37.6192 & -122.0158 & 4.76 & 3.23 \\
\hline $1997 / 11 / 29$ & $00: 31: 12.53$ & 38.7748 & -122.7520 & 1.89 & 1.20 \\
\hline $1997 / 12 / 13$ & $11: 51: 16.16$ & 37.2828 & -122.0525 & 5.68 & 2.97 \\
\hline $1997 / 12 / 23$ & $15: 11: 33.73$ & 37.5337 & -121.8008 & 8.37 & 1.50 \\
\hline $1998 / 01 / 17$ & $10: 00: 12.93$ & 37.8108 & -122.1928 & 4.49 & 2.40 \\
\hline $1998 / 02 / 05$ & $10: 00: 37.13$ & 38.7812 & -122.7477 & 4.31 & 1.10 \\
\hline & & & & & \\
\hline
\end{tabular}


Table 6: Events Recorded in the Study

\begin{tabular}{|l|l|l|l|l|l|}
\hline \multicolumn{1}{|c|}{ Earthquake } & \multicolumn{1}{|c|}{ Time } & \multicolumn{1}{c|}{ Latitude } & \multicolumn{1}{c|}{ Longitude } & \multicolumn{1}{c|}{ Depth } & Magnitude \\
\hline $1998 / 02 / 05$ & $12: 49: 15.58$ & 38.8555 & -122.8638 & 7.28 & 1.30 \\
\hline $1998 / 02 / 05$ & $17: 42: 20.63$ & 38.7915 & -122.7762 & 2.37 & 1.70 \\
\hline $1998 / 02 / 06$ & $14: 28: 24.04$ & 38.8035 & -122.8060 & 2.31 & 1.40 \\
\hline $1998 / 02 / 06$ & $17: 17: 21.10$ & 38.7810 & -122.7250 & 2.47 & 2.60 \\
\hline $1998 / 02 / 07$ & $17: 14: 50.24$ & 38.8267 & -122.8080 & 4.00 & 1.60 \\
\hline $1998 / 02 / 07$ & $20: 18: 24.25$ & 37.1395 & -121.5558 & 0.04 & 1.30 \\
\hline $1998 / 02 / 08$ & $18: 50: 48.90$ & 37.9643 & -122.0393 & 12.90 & 2.40 \\
\hline $1998 / 03 / 14$ & $10: 52: 27.96$ & 37.3777 & -122.2660 & 9.56 & 2.80 \\
\hline $1998 / 03 / 31$ & $11: 44: 09.90$ & 38.7748 & -122.7452 & 4.06 & 2.70 \\
\hline $1998 / 04 / 03$ & $19: 37: 45.14$ & 38.8898 & -123.0323 & 2.01 & 1.60 \\
\hline $1998 / 04 / 10$ & $04: 07: 49.46$ & 37.8670 & -122.2427 & 10.34 & 1.50 \\
\hline $1998 / 05 / 06$ & $11: 34: 37.96$ & 38.8055 & -122.7363 & 2.75 & 1.40 \\
\hline $1998 / 05 / 08$ & $08: 06: 30.49$ & 38.7985 & -122.7760 & 4.81 & 1.60 \\
\hline $1998 / 05 / 09$ & $04: 27: 07.86$ & 37.7557 & -122.5663 & 7.23 & 2.40 \\
\hline $1998 / 05 / 09$ & $10: 41: 24.35$ & 38.7823 & -122.7627 & 2.11 & 1.30 \\
\hline $1998 / 05 / 11$ & $08: 45: 17.54$ & 38.8162 & -122.8112 & 3.59 & 1.30 \\
\hline $1998 / 05 / 13$ & $10: 15: 19.21$ & 37.5627 & -122.3747 & 5.45 & 1.10 \\
\hline $1998 / 06 / 09$ & $02: 26: 43.61$ & 37.9127 & -122.2852 & 5.81 & 2.50 \\
\hline & & & & & \\
\hline
\end{tabular}

Table 7: Bay Bridge Site Recordings

\begin{tabular}{|l|l|l|l|l|l|l|l|l|}
\hline Earthquake & BBW2 & BBW5 & YBA & BE02 & BE07 & BE17 & BE23 & SFA \\
\hline \hline 9602042327 & & & & & & XXXX & & \\
\hline 9603200031 & & & & & XXXX & & & \\
\hline 9604120852 & & & & & XXXX & & & \\
\hline 9604141336 & & & & & XXXX & & & \\
\hline 9604240521 & & & & & XXXX & & & \\
\hline
\end{tabular}


Table 7: Bay Bridge Site Recordings

\begin{tabular}{|c|c|c|c|c|c|c|c|c|}
\hline Earthquake & BBW2 & BBW5 & YBA & BE02 & BE07 & BE17 & BE23 & SFA \\
\hline 9605141751 & & & & & $\mathrm{XXXX}$ & & & \\
\hline 9605150411 & & & & & $\mathrm{XXXX}$ & & & \\
\hline 9605212050 & $\mathrm{XXXX}$ & & & & & & & \\
\hline 9605300250 & & & & & XXXX & & & \\
\hline 9605310836 & & & & & $\mathrm{XXXX}$ & & & \\
\hline 9607010433 & & & & $\mathrm{XXXX}$ & $\mathrm{XXXX}$ & & & \\
\hline 9607041952 & & & & $\mathrm{XXXX}$ & & & & \\
\hline 9608252320 & & & & $\mathrm{XXXX}$ & & & & \\
\hline 9610312309 & & & & & & $\mathrm{XXXX}$ & & \\
\hline 9612241616 & & & & & & $\mathrm{XXXX}$ & & \\
\hline 9612310539 & & & & & & $\mathrm{XXXX}$ & & \\
\hline 9702050025 & $\mathrm{XXXX}$ & & & & $\mathrm{XXXX}$ & & & \\
\hline 9702141035 & & & & & $\mathrm{XXXX}$ & & & \\
\hline 9702171651 & & & & & $\mathrm{XXXX}$ & & & \\
\hline 9702201343 & & & & & $\mathrm{XXXX}$ & & & \\
\hline 9703110630 & $\mathrm{XXXX}$ & $\mathrm{XXXX}$ & & & $\mathrm{XXXX}$ & & & \\
\hline 9703110633 & $\mathrm{XXXX}$ & $\mathrm{XXXX}$ & & & & & & \\
\hline 9703280426 & & & & & $\mathrm{XXXX}$ & & & \\
\hline 9703300634 & & & & & $\mathrm{XXXX}$ & & & \\
\hline 9704031731 & & & & & $\mathrm{XXXX}$ & & & \\
\hline 9705021130 & $\mathrm{XXXX}$ & & & & & & & \\
\hline 9705021231 & $\mathrm{XXXX}$ & & & & & & & \\
\hline 9705291021 & & $\mathrm{XXXX}$ & & & & & & \\
\hline 9706190035 & & & & & & $\mathrm{XXXX}$ & & \\
\hline 9706190411 & & & & & & $\mathrm{XXXX}$ & & \\
\hline 9706250111 & & & & & & XXXX & & \\
\hline 9706250313 & & & & & & $\mathrm{XXXX}$ & & \\
\hline
\end{tabular}


Table 7: Bay Bridge Site Recordings

\begin{tabular}{|l|l|l|l|l|l|l|l|l|}
\hline Earthquake & BBW2 & BBW5 & YBA & BE02 & BE07 & BE17 & BE23 & SFA \\
\hline \hline 9707091614 & & & & XXXX & & XXXX & & \\
\hline 9707140611 & & & & & XXXX & & & \\
\hline 9707171946 & & & & XXXX & & & & \\
\hline 9708140853 & & XXXX & & XXXX & & XXXX & & \\
\hline 9708171841 & & & & & & XXXX & & \\
\hline 9708272029 & & & & & XXXX & & & \\
\hline 9708310024 & & $\mathrm{XXXX}$ & & & $\mathrm{XXXX}$ & $\mathrm{XXXX}$ & & \\
\hline 9708311718 & & & & & $\mathrm{XXXX}$ & & & \\
\hline 9709031900 & & & & & $\mathrm{XXXX}$ & & & \\
\hline 9709080010 & & & & & & $\mathrm{XXXX}$ & & \\
\hline 9709122119 & & & & & & $\mathrm{XXXX}$ & & \\
\hline 9709240441 & & $\mathrm{XXXX}$ & & & & & & \\
\hline 9710190523 & & & & & $\mathrm{XXXX}$ & & & \\
\hline 9710271430 & $\mathrm{XXXX}$ & $\mathrm{XXXX}$ & & $\mathrm{XXXX}$ & & & & \\
\hline 9711151551 & & & & & $\mathrm{XXXX}$ & & & \\
\hline 9711192105 & & $\mathrm{XXXX}$ & & $\mathrm{XXXX}$ & & & & \\
\hline 9711290031 & & & & & & $\mathrm{XXXX}$ & & \\
\hline 9712131151 & $\mathrm{XXXX}$ & & & & $\mathrm{XXXX}$ & & & \\
\hline 9712231511 & & & & & & $\mathrm{XXXX}$ & & \\
\hline 9801171000 & $\mathrm{XXXX}$ & $\mathrm{XXXX}$ & & & $\mathrm{XXXX}$ & $\mathrm{XXXX}$ & & \\
\hline 9802051000 & & & & & $\mathrm{XXXX}$ & & & \\
\hline 9802051249 & & & & & $\mathrm{XXXX}$ & & & \\
\hline 9802051742 & & & & & $\mathrm{XXXX}$ & & & \\
\hline 9802061428 & & & & & & $\mathrm{XXXX}$ & & \\
\hline 9802061717 & & & & & $\mathrm{XXXX}$ & & & \\
\hline 9802071714 & & & & & $\mathrm{XXXX}$ & & & \\
\hline 9802072018 & & & & & & $\mathrm{XXXX}$ & & \\
\hline
\end{tabular}


Table 7: Bay Bridge Site Recordings

\begin{tabular}{|l|l|l|l|l|l|l|l|l|}
\hline Earthquake & BBW2 & BBW5 & YBA & BE02 & BE07 & BE17 & BE23 & SFA \\
\hline \hline 9802081850 & & & & & XXXX & XXXX & & \\
\hline 9803141052 & XXXX & & & & & & & \\
\hline 9803311144 & & & & & XXXX & & & \\
\hline 9804031937 & & & & & XXXX & & & \\
\hline 9804100407 & & & & & & XXXX & & \\
\hline 9805061134 & & & & & & & & XXXX \\
\hline 9805080806 & & & & & & & & XXXX \\
\hline 9805090427 & $\mathrm{XXXX}$ & & & & & & & \\
\hline 9805091041 & & & & & & & & $\mathrm{XXXX}$ \\
\hline 9805110845 & & & & & & & & $\mathrm{XXXX}$ \\
\hline 9805131015 & & & & & & & & $\mathrm{XXXX}$ \\
\hline 9806090226 & $\mathrm{XXXX}$ & & & & $\mathrm{XXXX}$ & & & \\
\hline
\end{tabular}

Table 8: Dumbarton Bridge Site Recordings

\begin{tabular}{|c|c|c|c|}
\hline Earthquake & DB01 & DB27 & DB44 \\
\hline 9703110630 & & $\mathrm{XXXXXXXXXX}$ & $\mathrm{XXXXXXXXXX}$ \\
\hline 9703110632 & & XXXXXXXXXX & $\mathrm{XXXXXXXXXX}$ \\
\hline 9703110633 & & XXXXXXXXXX & $\mathrm{XXXXXXXXXX}$ \\
\hline 9703110641 & & XXXXXXXXXX & XXXXXXXXXX \\
\hline 9703181350 & & $\mathrm{XXXXXXXXXX}$ & \\
\hline 9703271130 & & $\mathrm{XXXXXXXXXX}$ & $\mathrm{XXXXXXXXXX}$ \\
\hline 9703271338 & & XXXXXXXXXX & $\mathrm{XXXXXXXXXX}$ \\
\hline 9703271539 & & $\mathrm{XXXXXXXXXX}$ & $\mathrm{XXXXXXXXXX}$ \\
\hline 9703272247 & & & 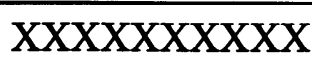 \\
\hline 9703280426 & & $\mathrm{XXXXXXXXXX}$ & $\mathrm{XXXXXXXXXX}$ \\
\hline 9704010136 & & XXXXXXXXXX & XXXXXXXXXX \\
\hline
\end{tabular}


Table 8: Dumbarton Bridge Site Recordings

\begin{tabular}{|c|c|c|c|}
\hline Earthquake & DB01 & DB27 & DB44 \\
\hline 9704060741 & $\mathrm{XXXXXXXXXX}$ & & \\
\hline 9704141029 & $\mathrm{XXXXXXXXXX}$ & & \\
\hline 9704221118 & & $\mathrm{XXXXXXXXXX}$ & $\mathrm{XXXXXXXXXX}$ \\
\hline 9705010038 & & $\mathrm{XXXXXXXXXX}$ & \\
\hline 9705010945 & & & $\mathrm{XXXXXXXXXX}$ \\
\hline 9705020902 & & XXXXXXXXXX & \\
\hline 9705021130 & & $\mathrm{XXXXXXXXXX}$ & \\
\hline 9705021231 & & $\mathrm{XXXXXXXXXX}$ & \\
\hline 9705200922 & & $\mathrm{XXXXXXXXXX}$ & \\
\hline 9705291021 & & $\mathrm{XXXXXXXXXX}$ & \\
\hline 9705291828 & & XXXXXXXXXX & \\
\hline 9706221626 & & XXXXXXXXXX & \\
\hline 9707040259 & & $\mathrm{XxXXXXXXXX}$ & \\
\hline 9707091614 & & $\mathrm{XXXXXXXXXX}$ & \\
\hline 9707110831 & & $\mathrm{XXXXXXXXXX}$ & \\
\hline 9707140611 & & XXXXXXXXXX & \\
\hline 9707301130 & & $\mathrm{XXXXXXXXXX}$ & \\
\hline 9708122201 & & XXXXXXXXXX & \\
\hline 9708140853 & & $\mathrm{XXXXXXXXXX}$ & \\
\hline 9708231426 & & $\mathrm{XXXXXXXXXX}$ & \\
\hline 9708310024 & & XXXXXXXXXX & \\
\hline 9709180425 & & $\mathrm{XXXXXXXXXX}$ & \\
\hline 9709230952 & & XXXXXXXXXX & \\
\hline 9709261958 & & $\mathrm{XXXXXXXXXX}$ & \\
\hline 9710270029 & & 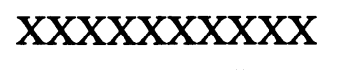 & \\
\hline 9710271430 & & $\mathrm{XXXXXXXXXX}$ & \\
\hline 9711192105 & & $\mathrm{XXXXXXXXXX}$ & \\
\hline
\end{tabular}




\section{San Francisco Bay Bridges}

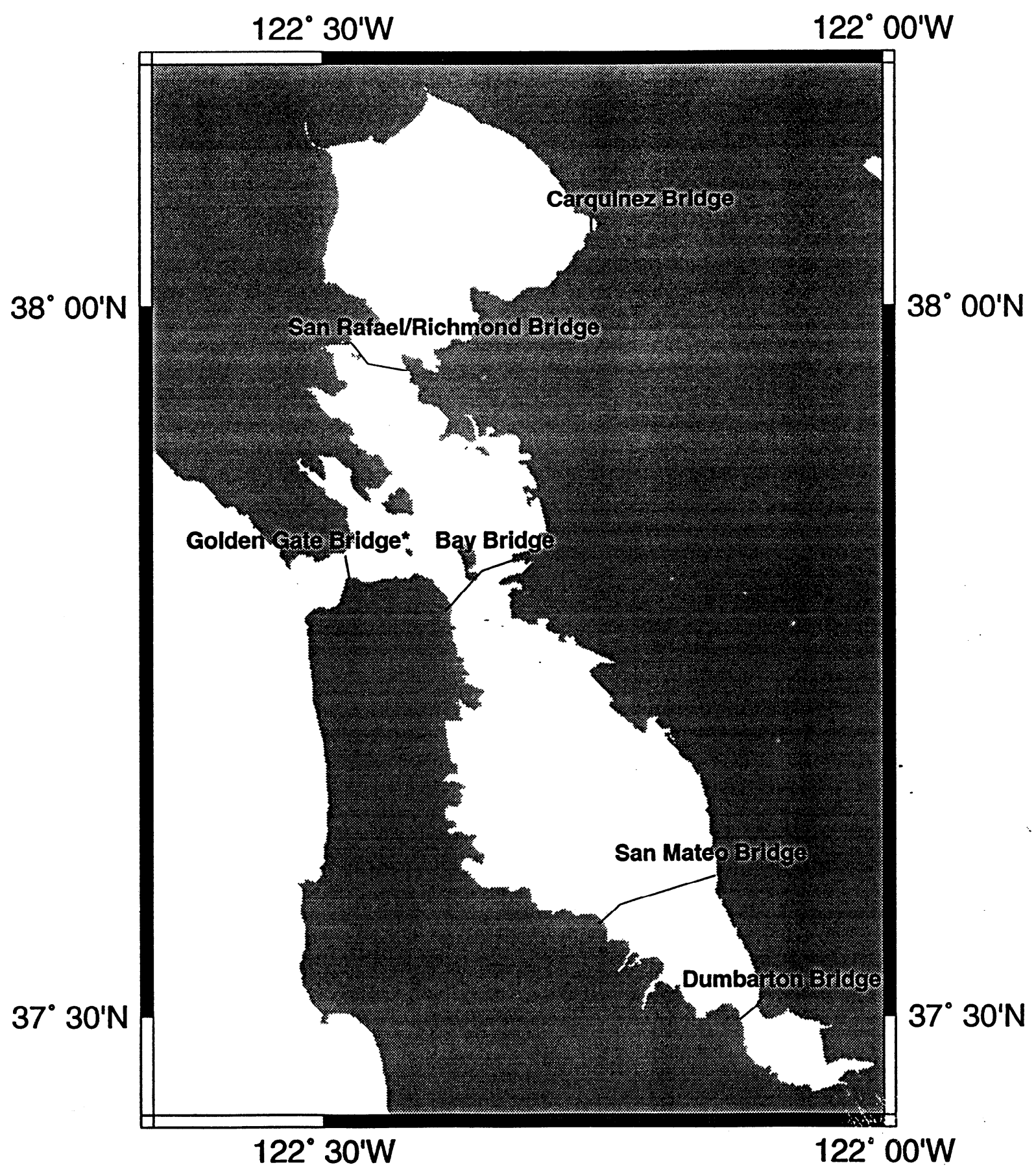

Figure 1: Map of the San Francisco Bay. (*Bridge not used in the bridge network study) 
Dumbarton Bridge
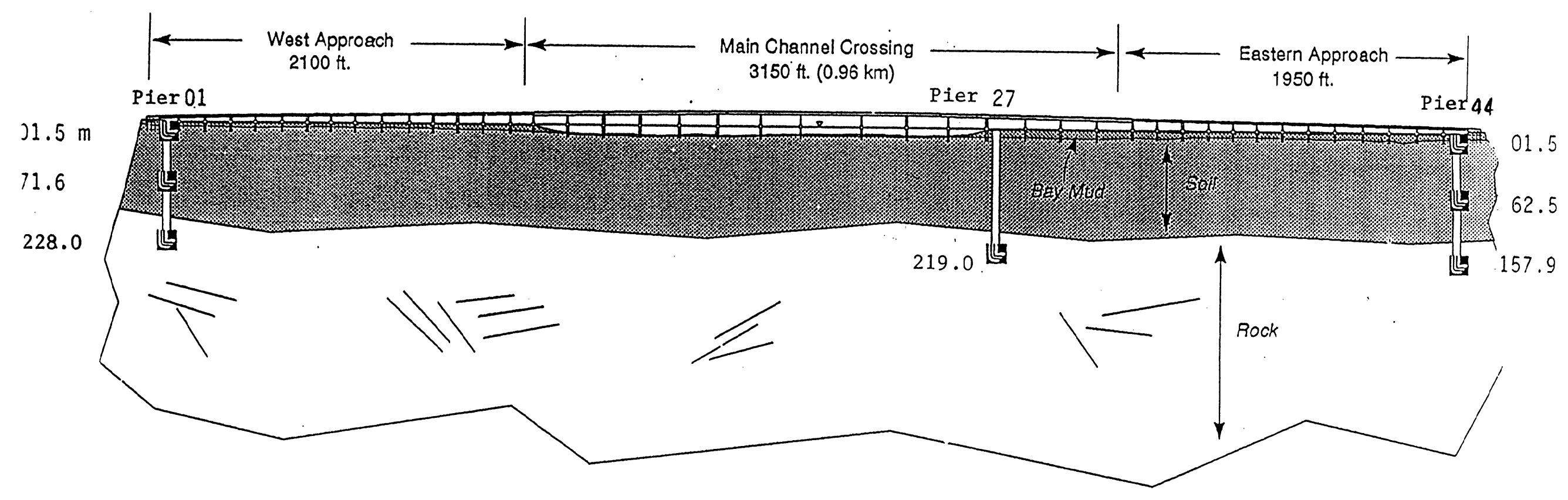

Figure 2: Location of the LLNL seismic instrumentation along the Dumbarton Bridge. 

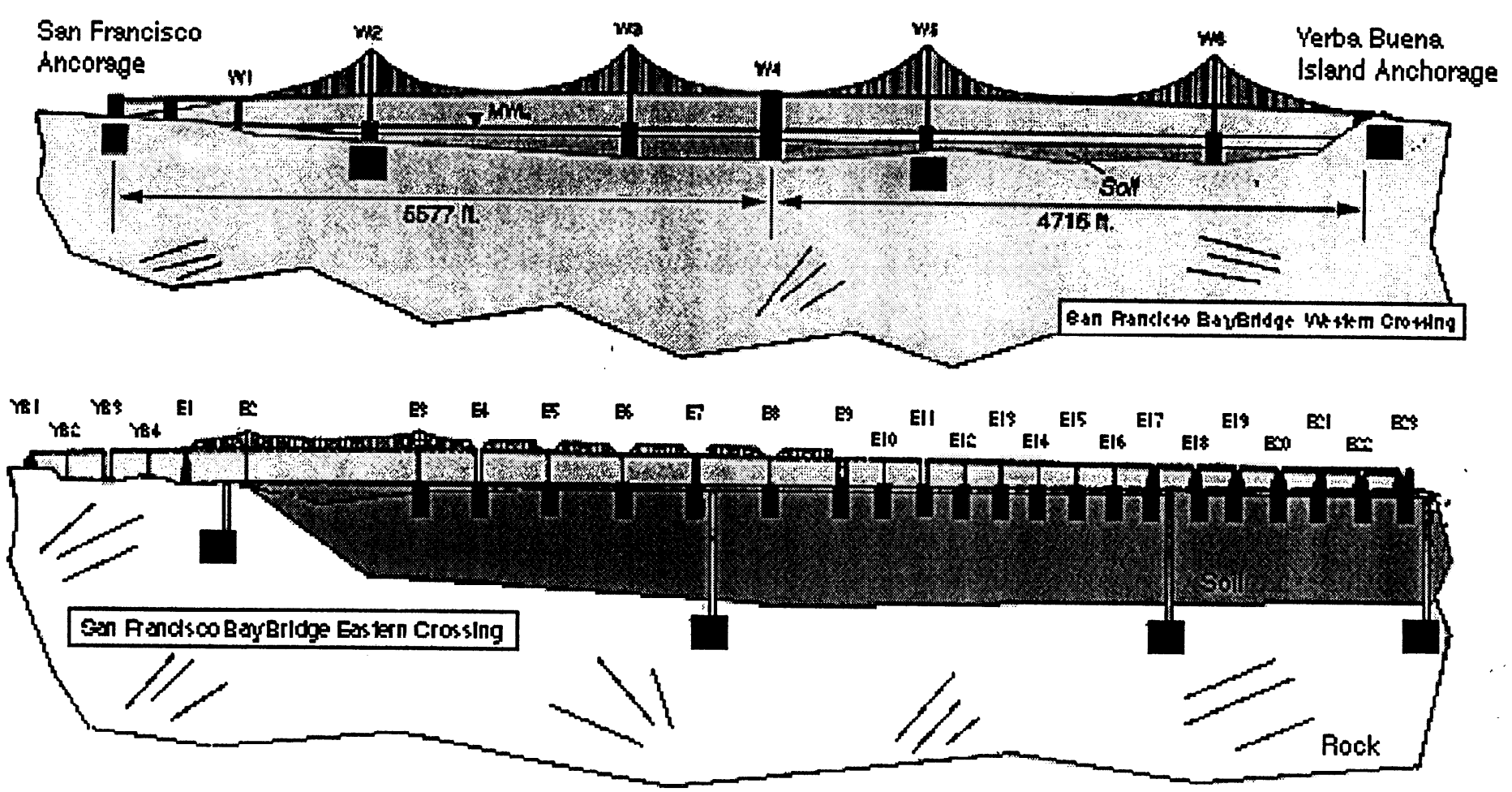

Figure 3: Location of LLNL/UC Berkeley seismic instrumentation along the San Francisco- Oakland Bay. Bridge. 


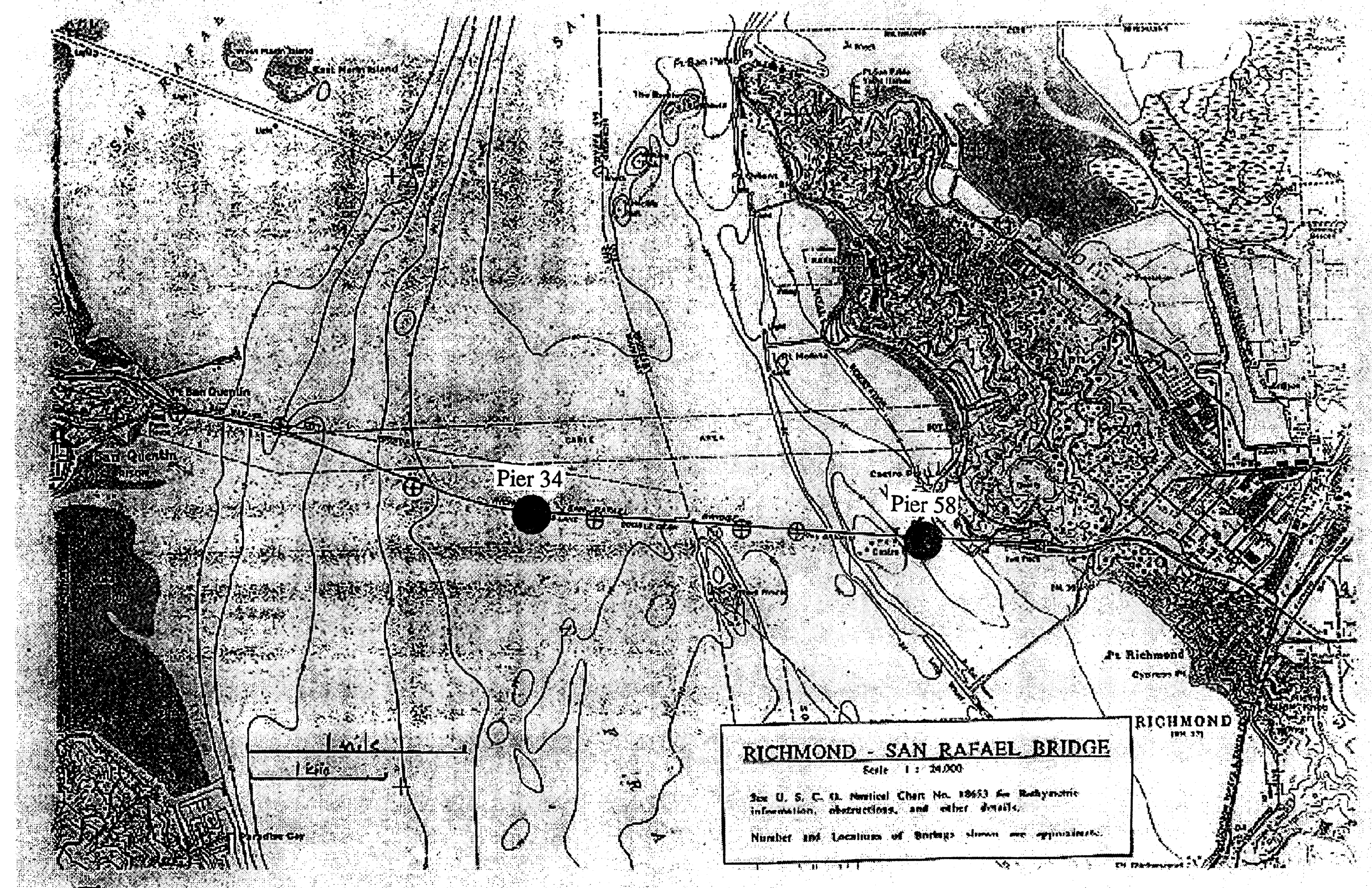

Figure 4: Location of seismic instrumentation along the Richmond- San Rafael Bridge. 


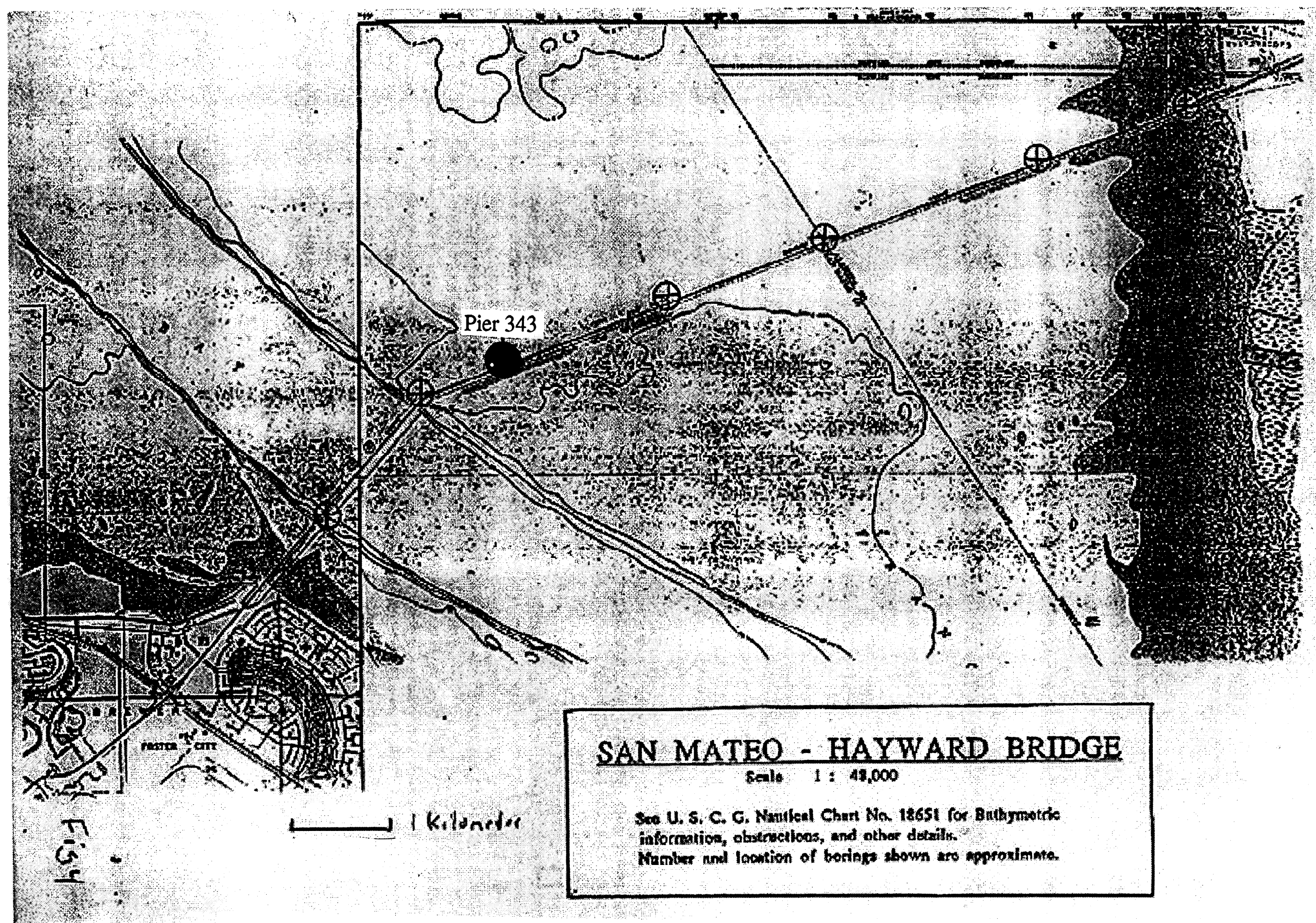

Figure 5: Location of seismic instrumentation along the San Maten- Hayward Bridge. 


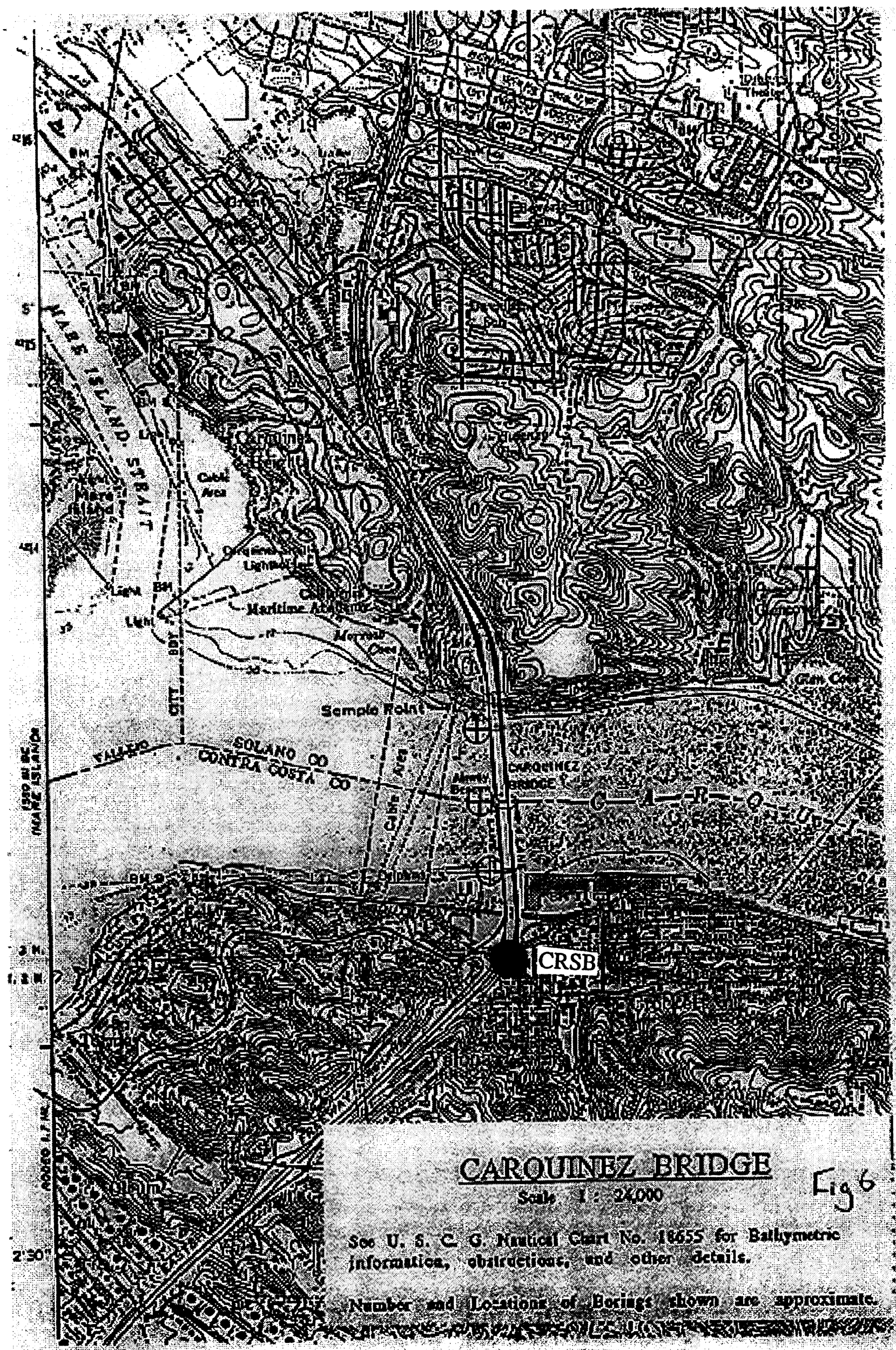

Figure 6: Location of seismic instrumentation along the Carqueniz Bridge. 


\section{Bay Bridge Recordings}

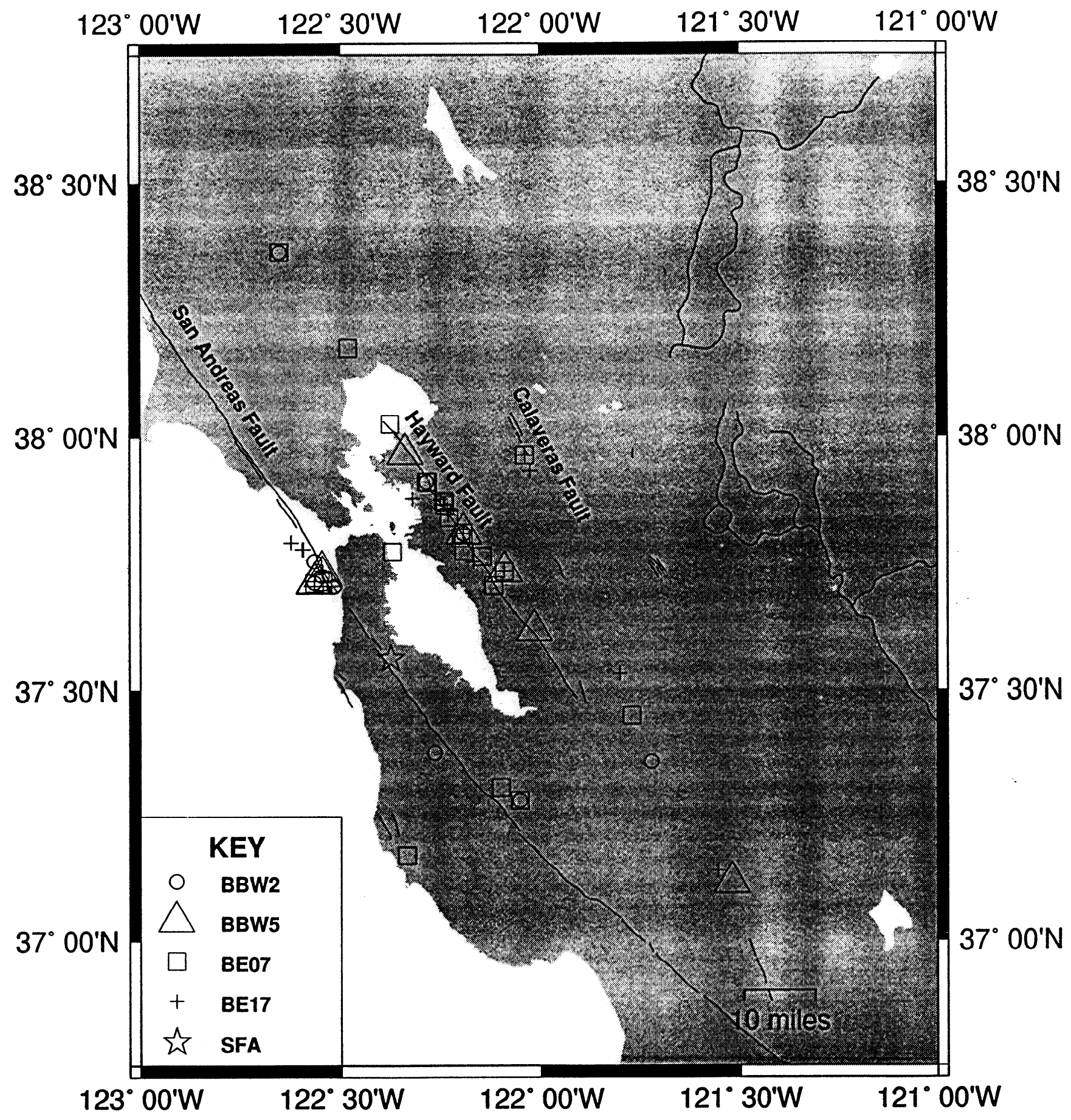

GMT Aug 24 14:50 Figure 7: Plot of earthquakes recorded by the Bay Bridge recorders. 


\section{Dumbarton Bridge Recordings}

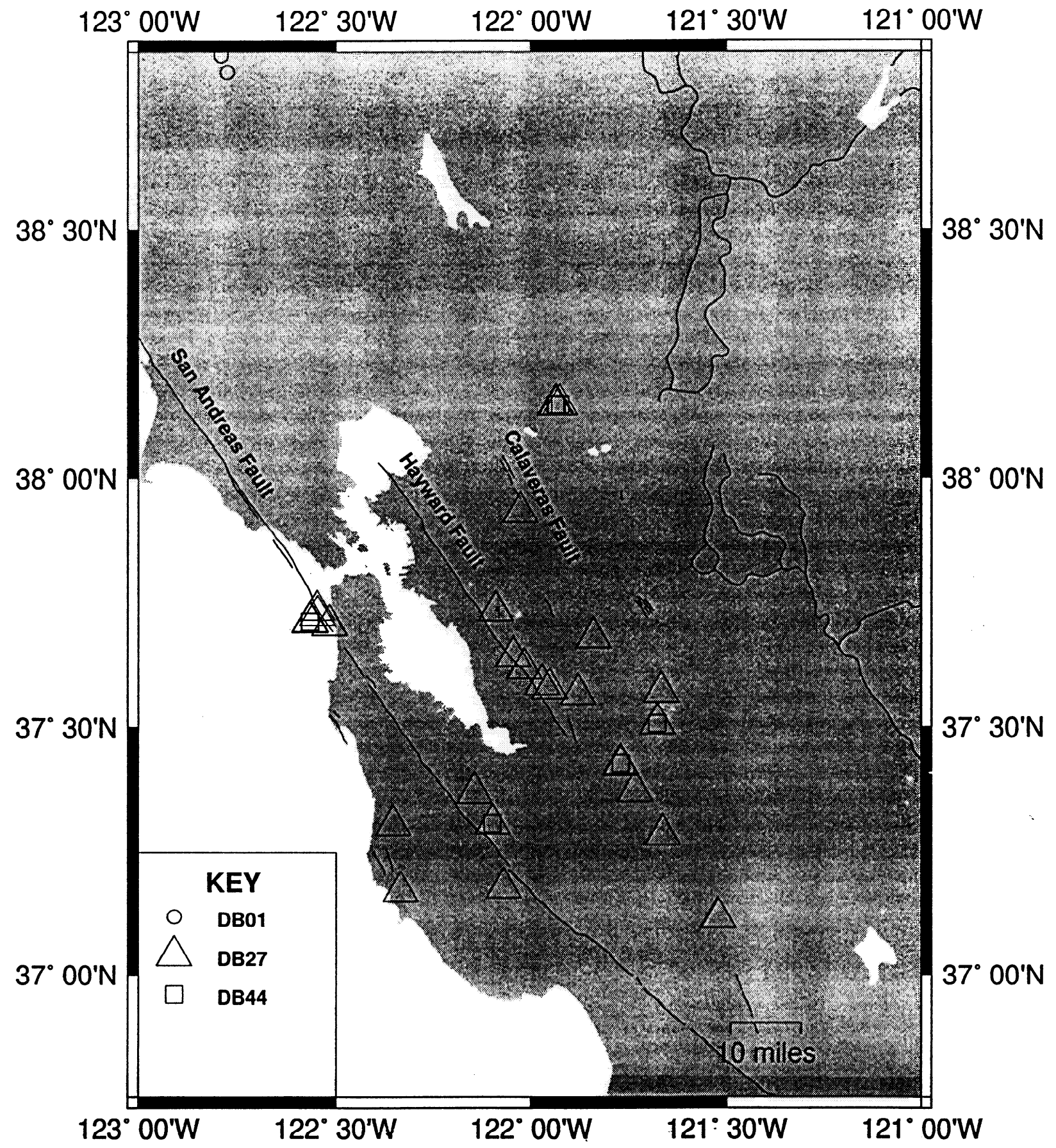

GMT Aug 24 18:27 Figure 8: Plot of earthquakes recorded by the Dumbarton Bridge recorders. 


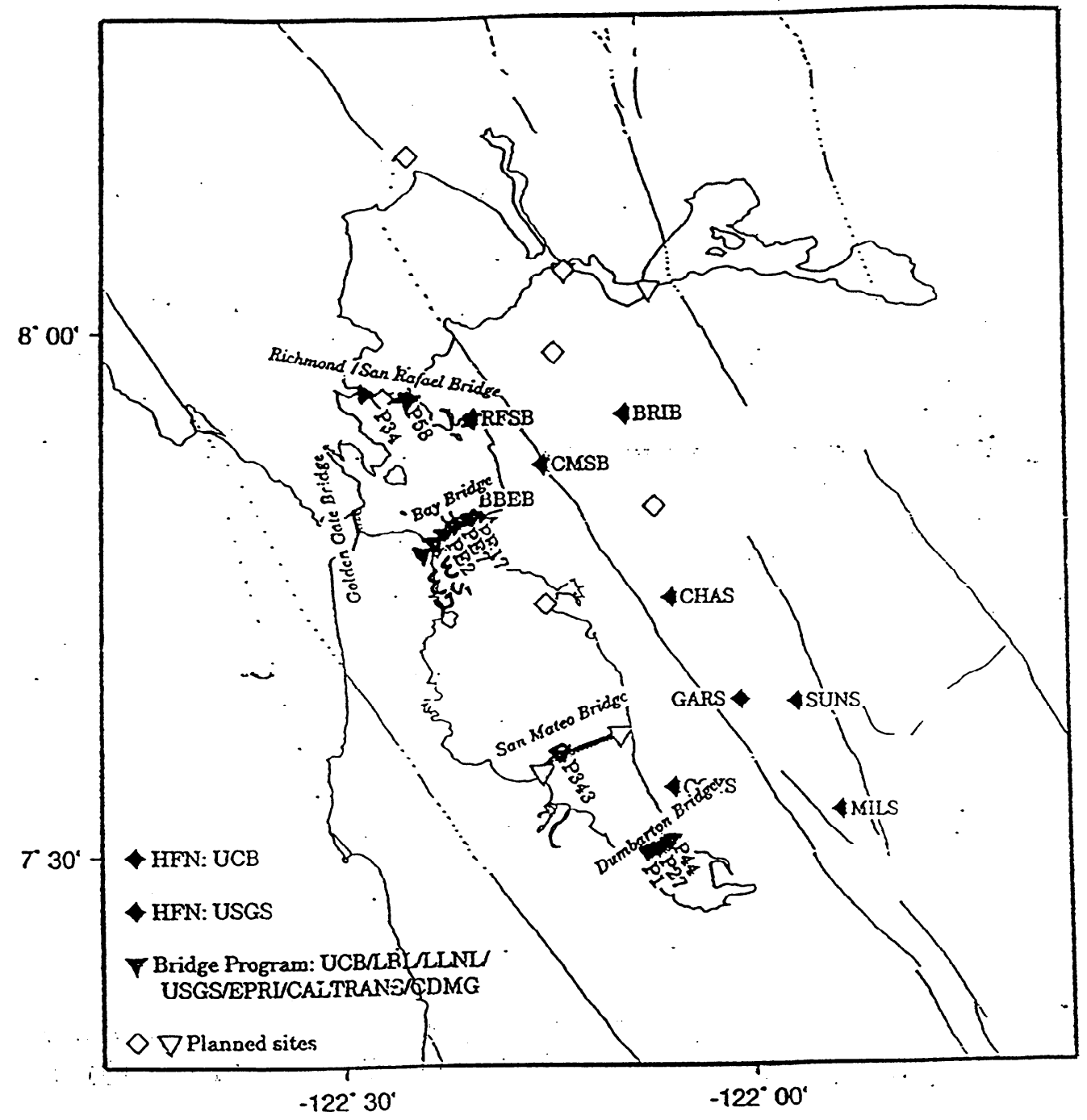

Figure 9: Locations of Bay Area Borehole Network. Instruments are placed 100' into bedrock. 

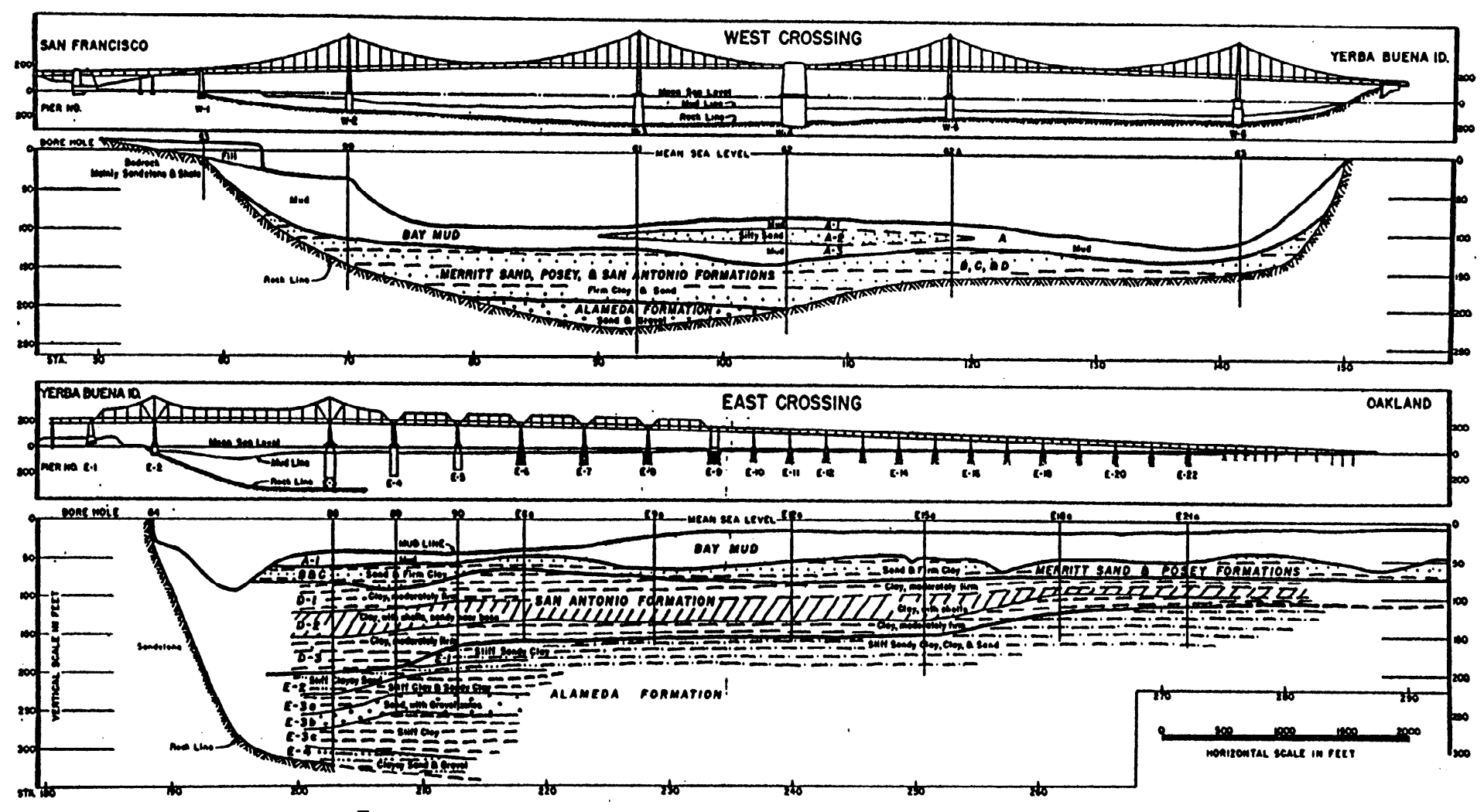

Figure 10: Subsurface geology beneath the San Francisco- Oakland Bay Bridge as inferred by Trask and Rolston (1951) and later updated by Rogers and Figures, 1991, Engineering Geologic Site Characterization of the Greater Oakland-Alameda Area, Alameda and San Francisco Counties, California : NSF Report, p. 46. 


\section{Depth to Bedrock}

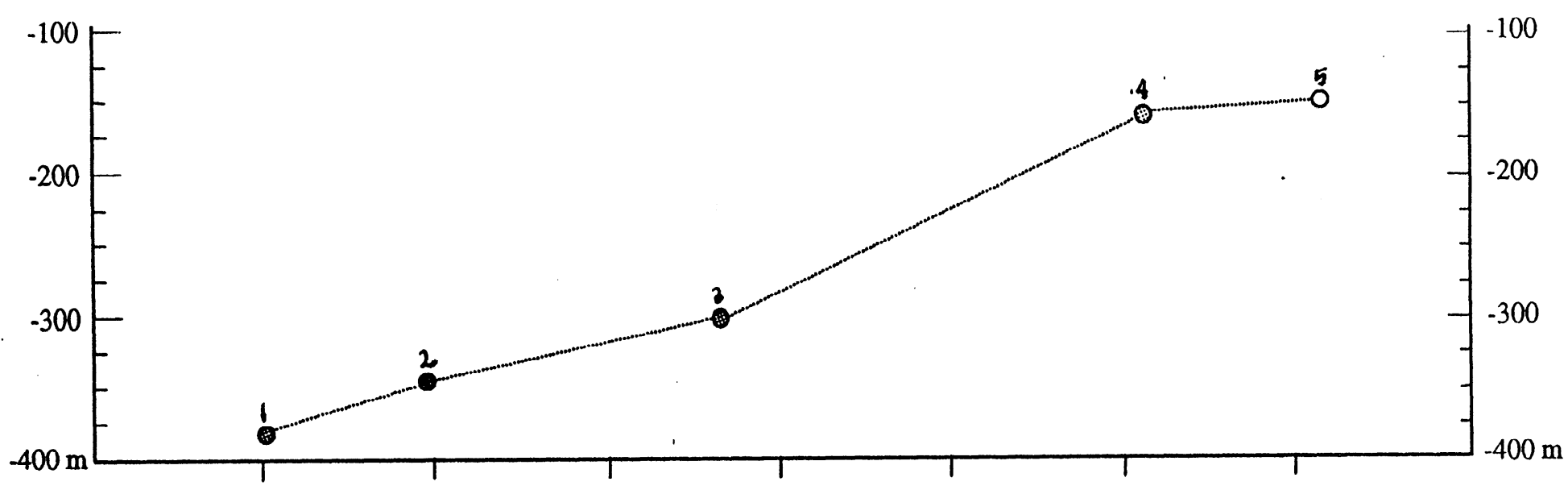

Figure 11: Depth to bedrock at lowest elevation under each bridge marking the bottom of the Alameda formation.

\begin{tabular}{|c|c|c|c|}
\hline $\begin{array}{c}\text { Golden Gate } \\
\text { Bridge }\end{array}$ & $\dot{0}$ & $\begin{array}{l}\text { Carquinez } \\
\text { Bridge }\end{array}$ & १ิ \\
\hline $\begin{array}{c}\text { San Francisco- } \\
\text { Oakland Bay Bridge }\end{array}$ & 2 & $\begin{array}{l}\text { Benica- } \\
\text { Martinez Bridge }\end{array}$ & 5 \\
\hline $\begin{array}{c}\text { Richmond- } \\
\text { San Rafael Bridge }\end{array}$ & 3 & $1: 10,000$ & \\
\hline
\end{tabular}



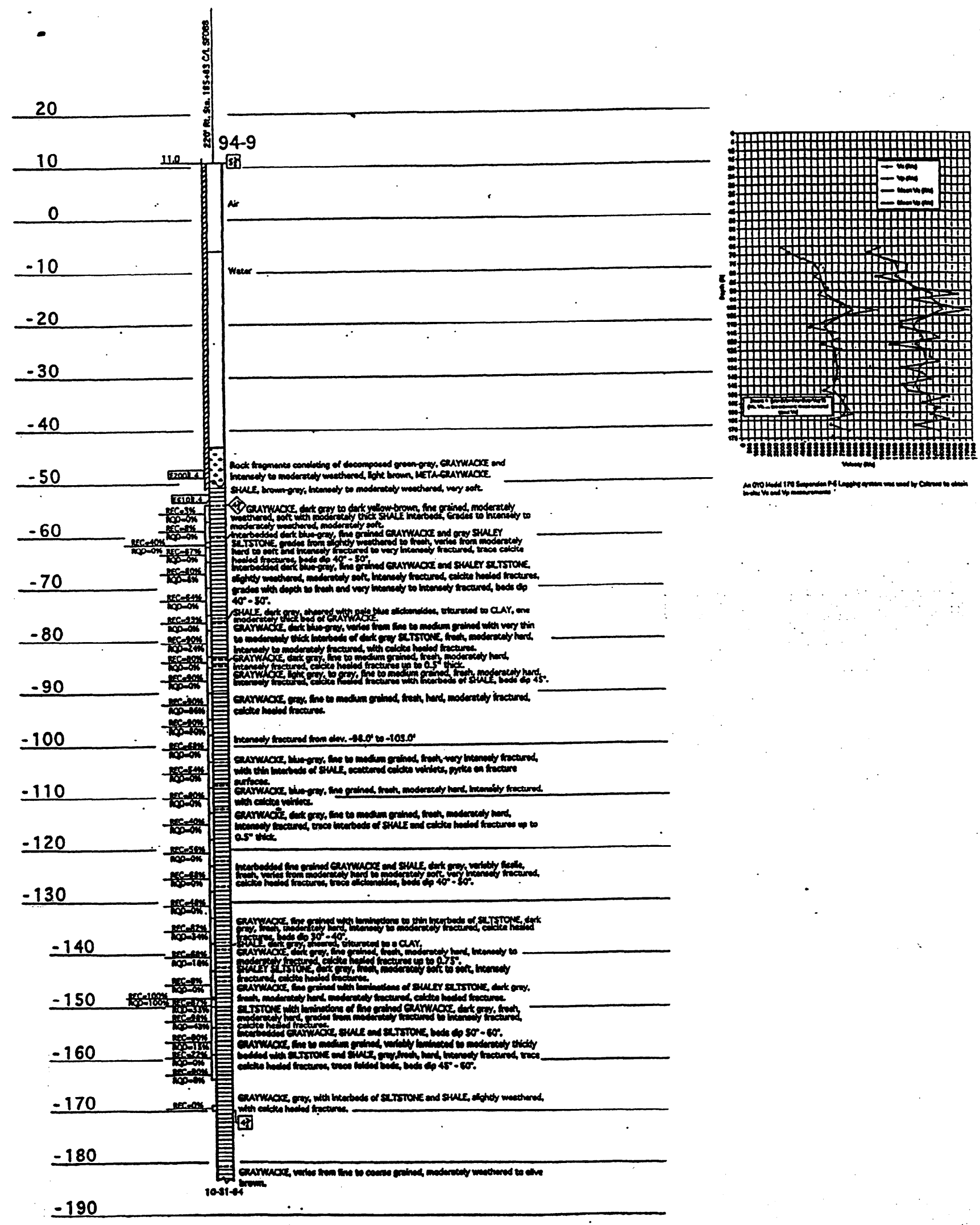

Figure 12: Caltrans borehole log for 94-9, between Pier E-1 and E-2, on the Bay Bridge. 


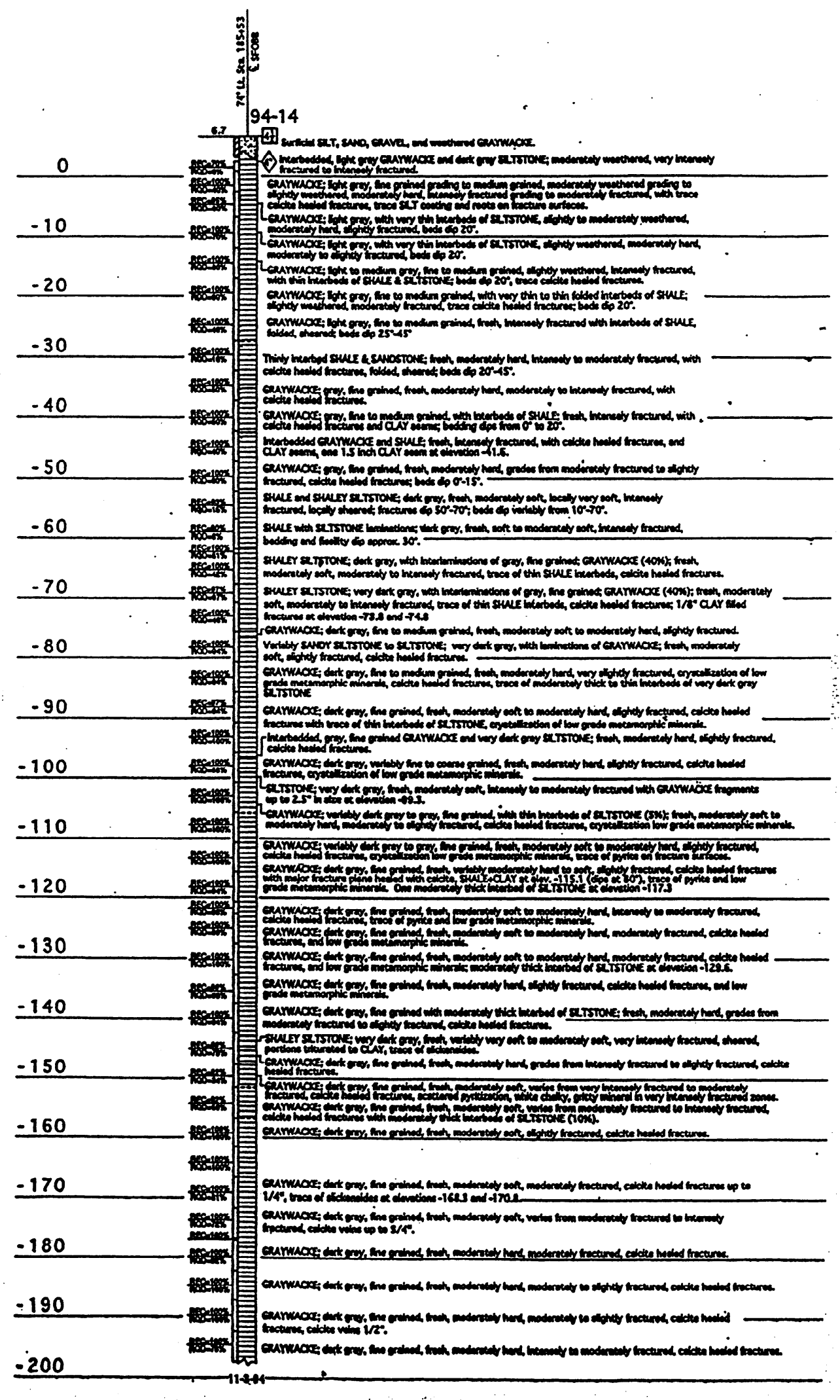

Figure 13: Caltrans borehole log for $94-14$, between pier E-1 and E-2, on the Bay Bridge. 


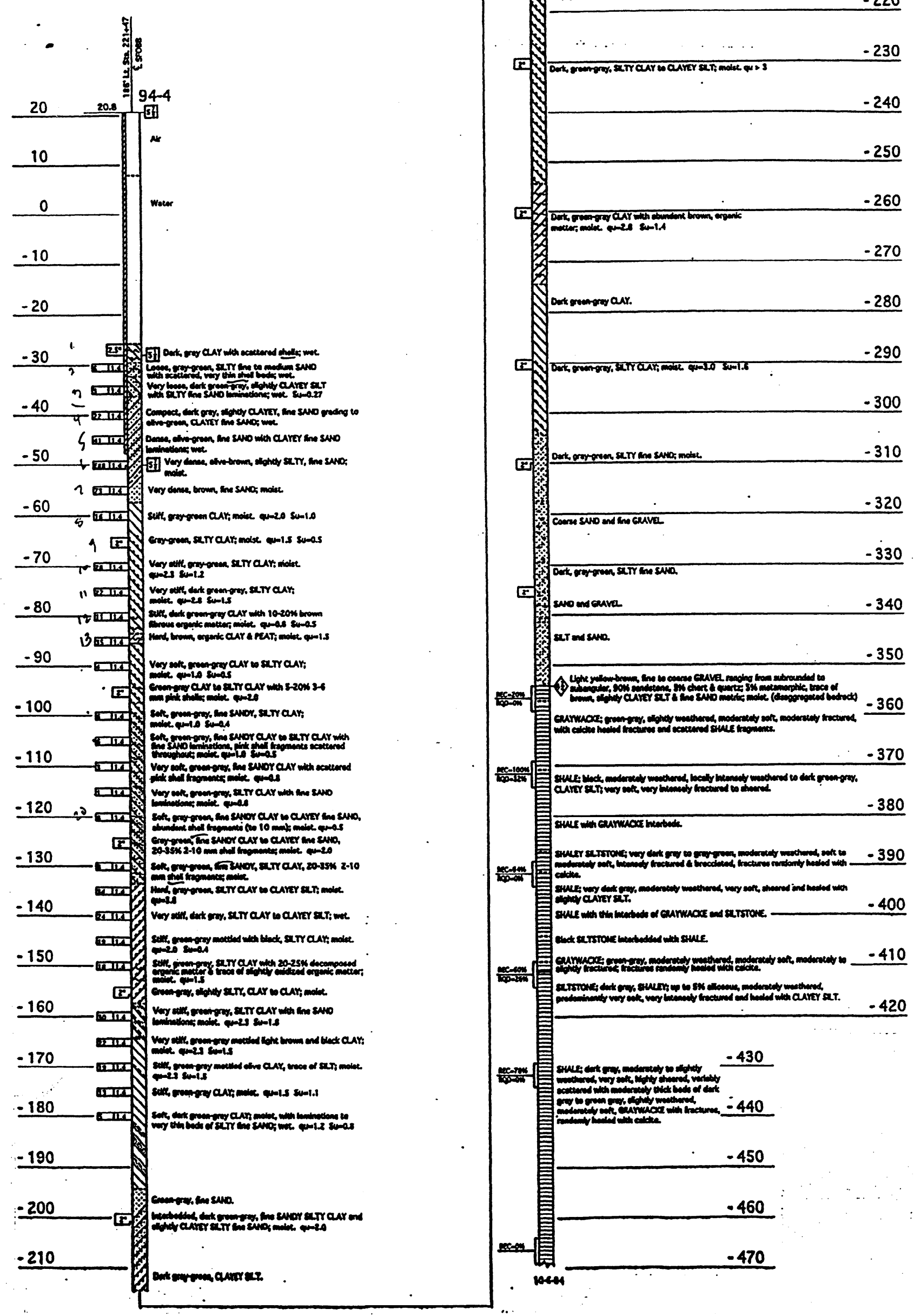

Figure 14: Caltrans borehole for 94-4, pier E-7, on the Bay Bridge. 


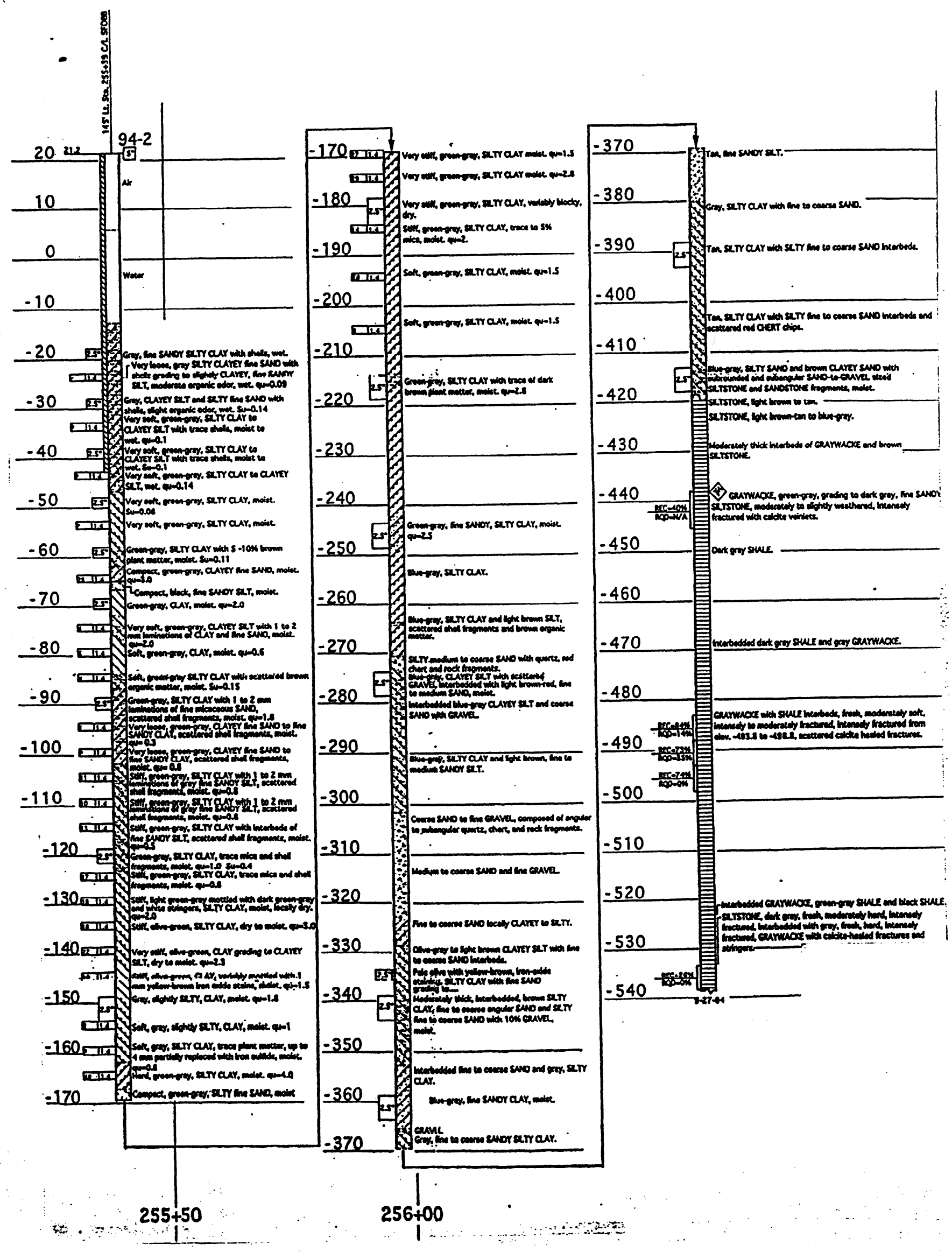

Figure 15: Caltrans borehole log for 94-2, Pier E-17, on the Bay Bridge. 


\section{Dumbarton Bridge Seismic Retrofit, Boring 93B11R Downhole Interval Velocities}

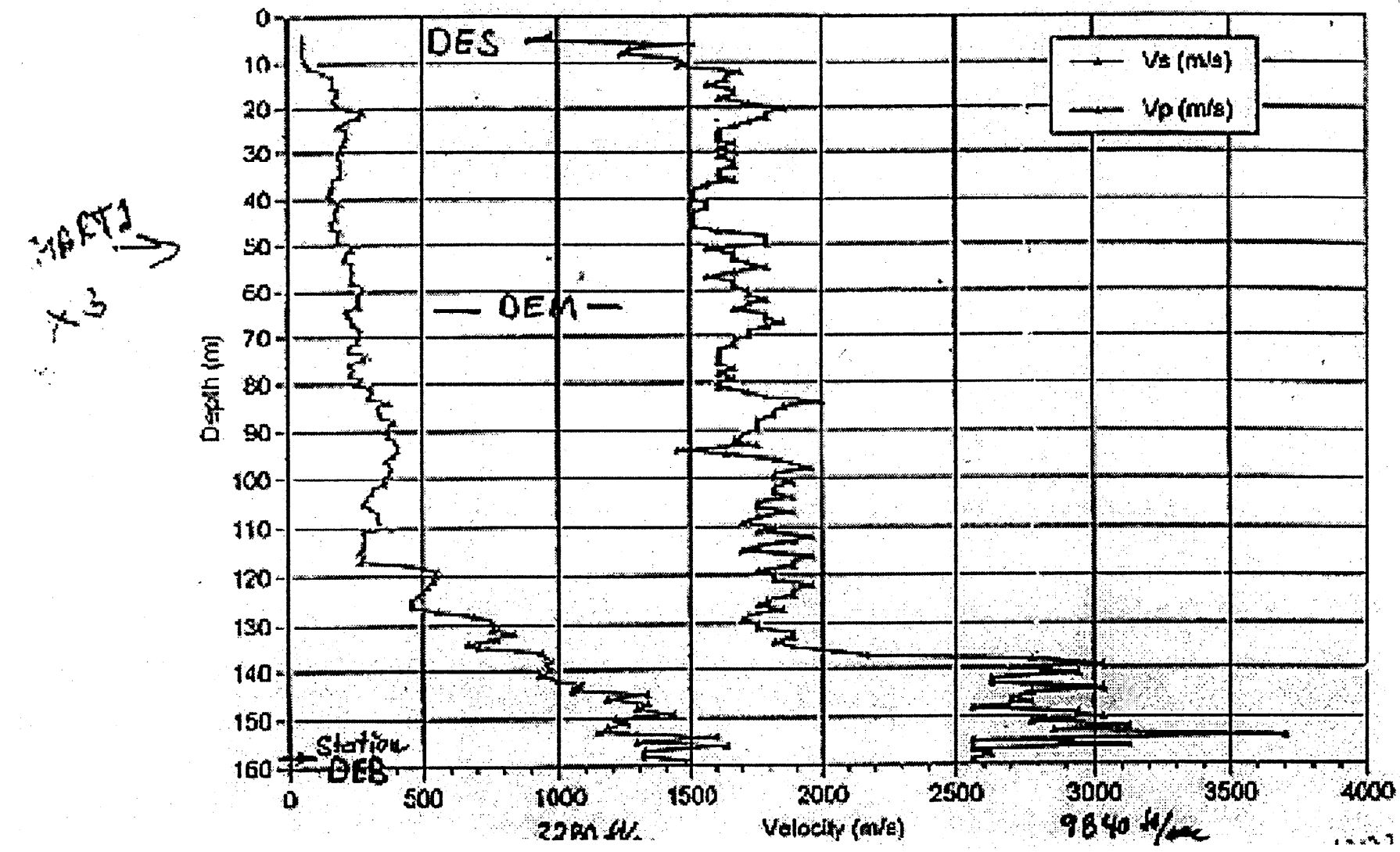

Figure 16: Velocity profile for deep hole at the west end, pile 1, of the Dumbarton Bridge. 


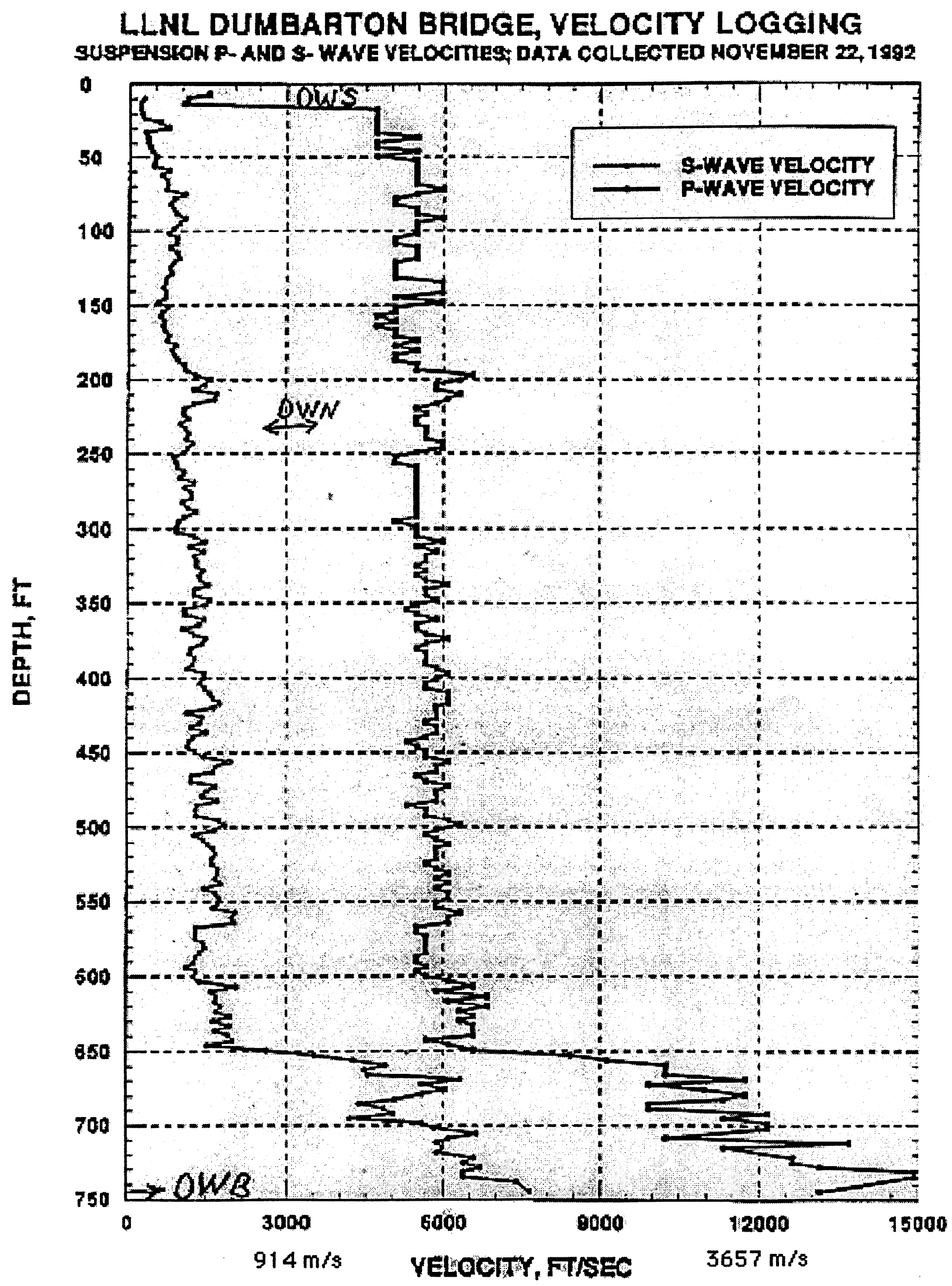

Figure 17: Velocity profile for deep hole at fishing pier, pile 27, of the Dumbarton Bridge. 


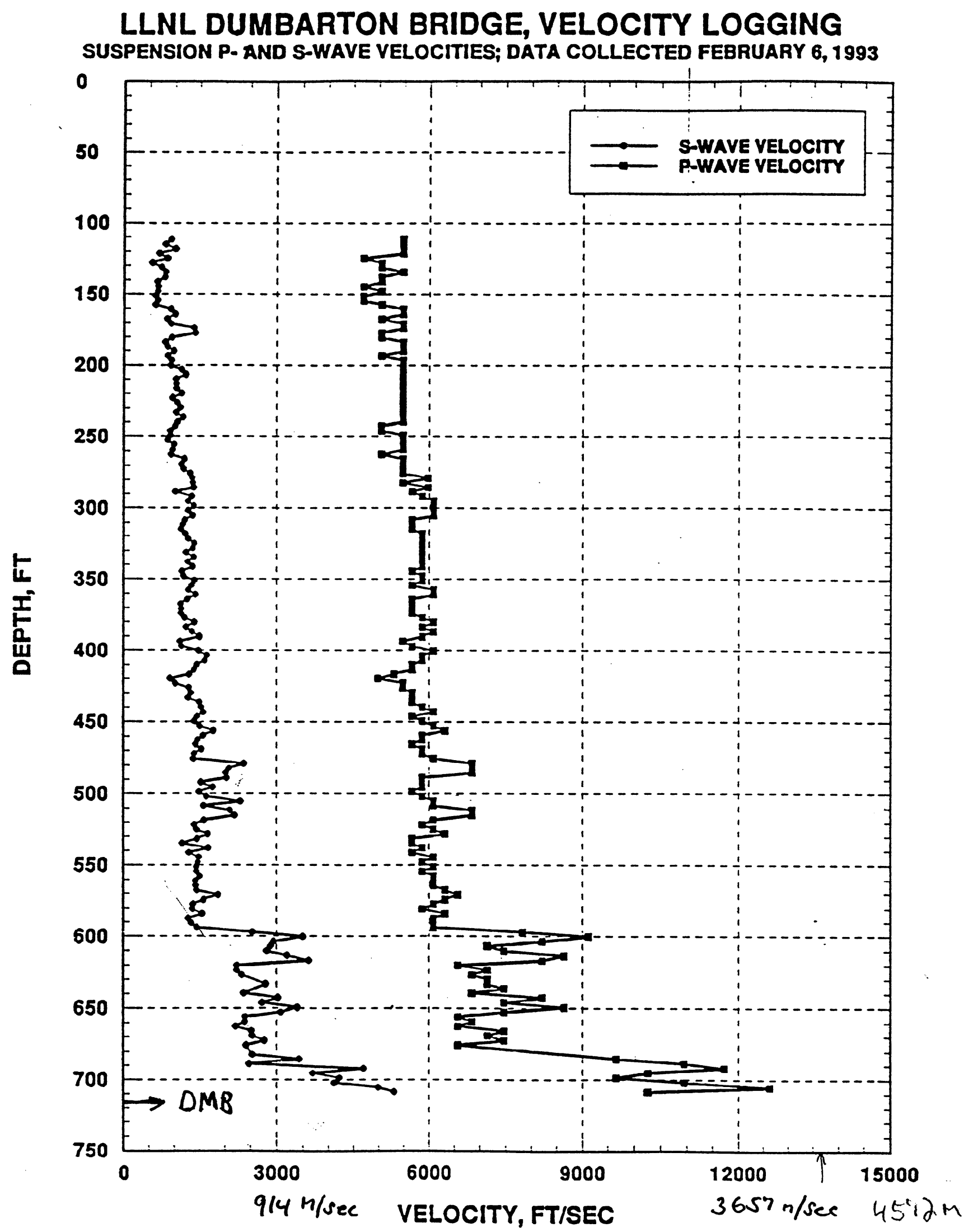

Figure 18: Velocity profile for deep hole on the east end, pile 44, of the Dumbarton Bridge. 
Figure 19: Recordings of acceleration at each of the deep bore sensors along the

Dumbarton Bridge (north component only) from a $M=1.9$ earthquake located $19 \mathrm{~km}$ to the west, on the San Andreas fault.

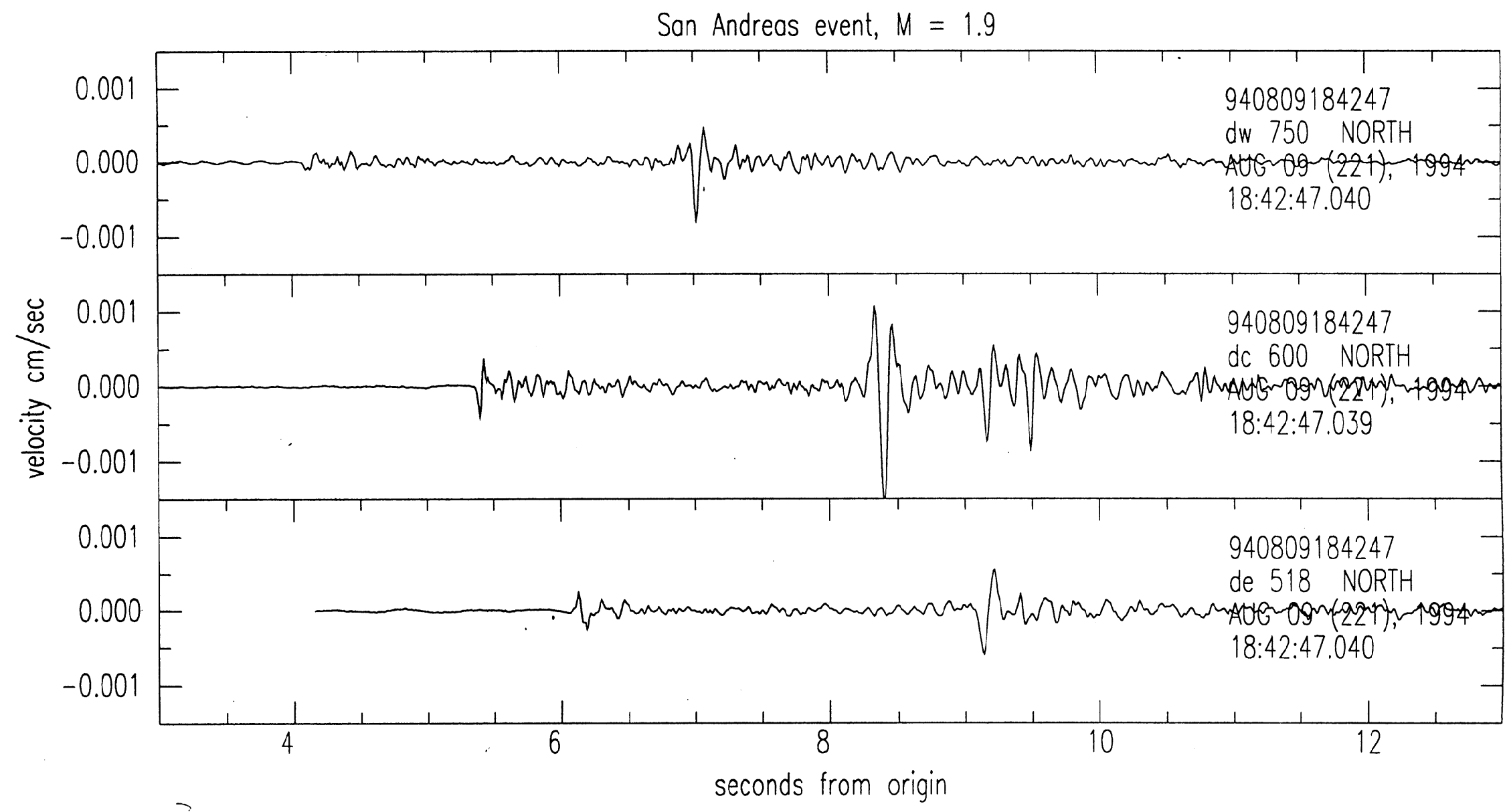




\section{Top and Bottom Borehole Recordings}

- linear response amplification

- $M=6.5$ event at $430 \mathrm{Km}$

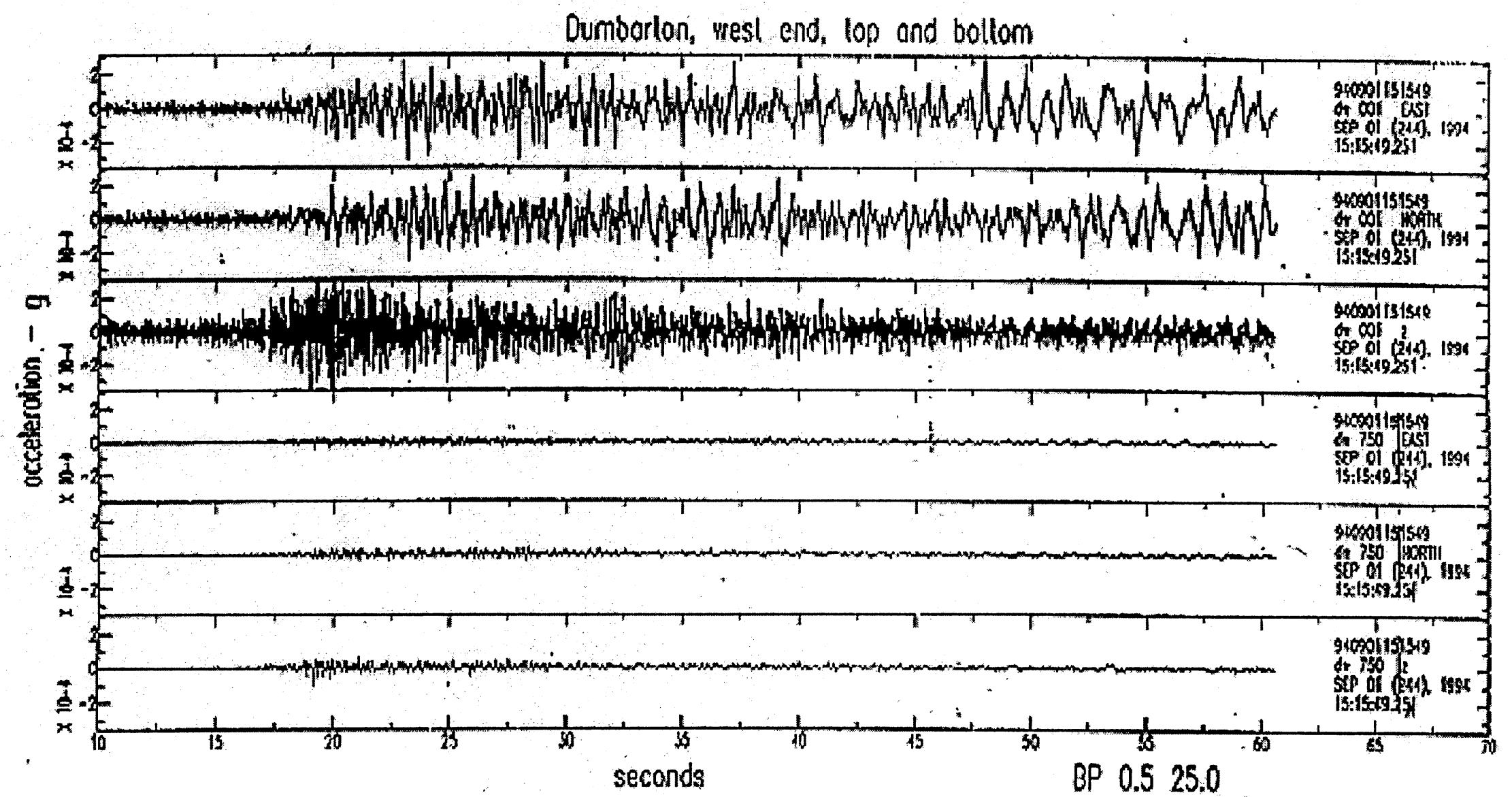

Figure 20: Accelerograms from the top and bottom sensor pairs for a $M=6.5$ located 490)

$\mathrm{km}$ distant, near the Mendocino triple junction. 


\section{Top, Middle and Bottom Borehole Amplification}

- Fourier amplitude spectra

- rock is highly competent graywacke

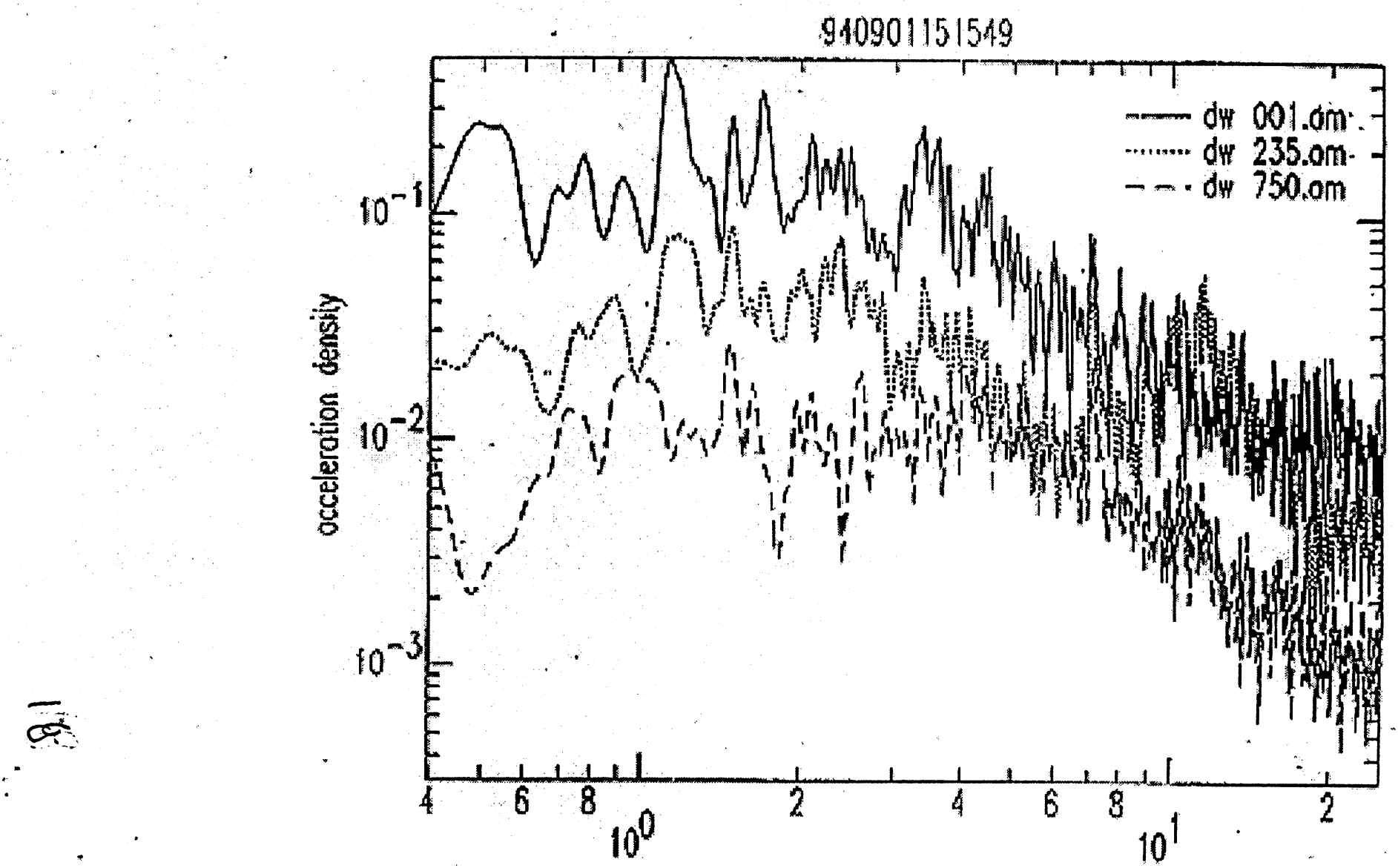

Figure 21: Spectra analysis of the eastern component from the top, middle, and bottom of the borehole on the Dumbarton Bridge. 
August 11, 1993 event; Calaveras Fault; $M=4.9 ; D=45 \mathrm{~km}$

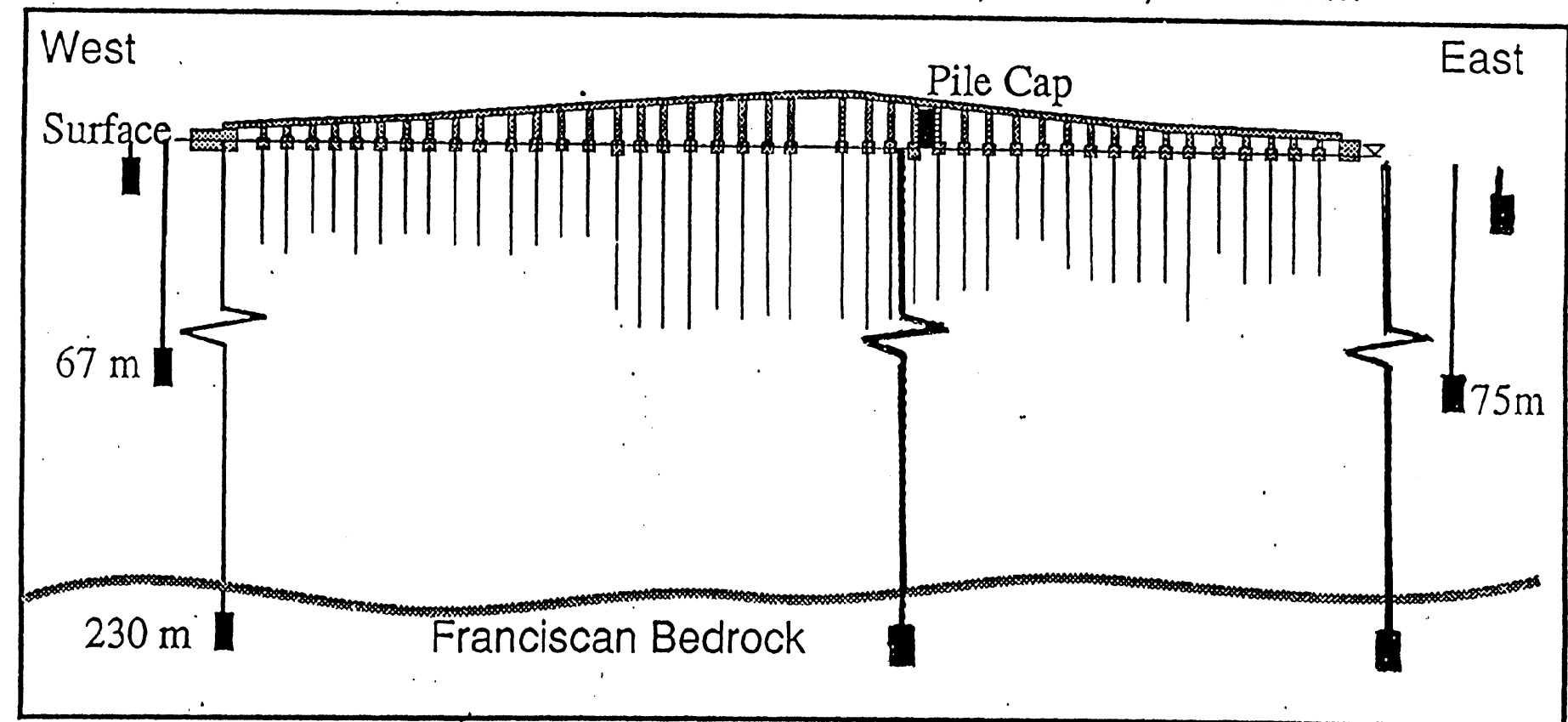

2

Horizontal Velocity transverse to the bridge $(\mathrm{cm} / \mathrm{sec})$

$230 \mathrm{~m}$

Dnax $=0.017$

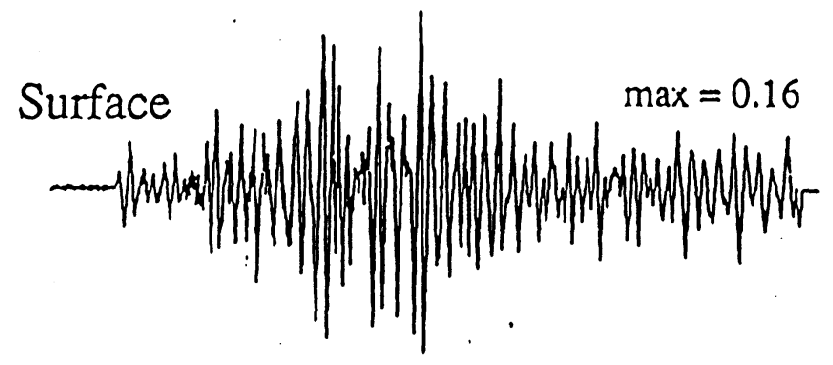

50 Seconds

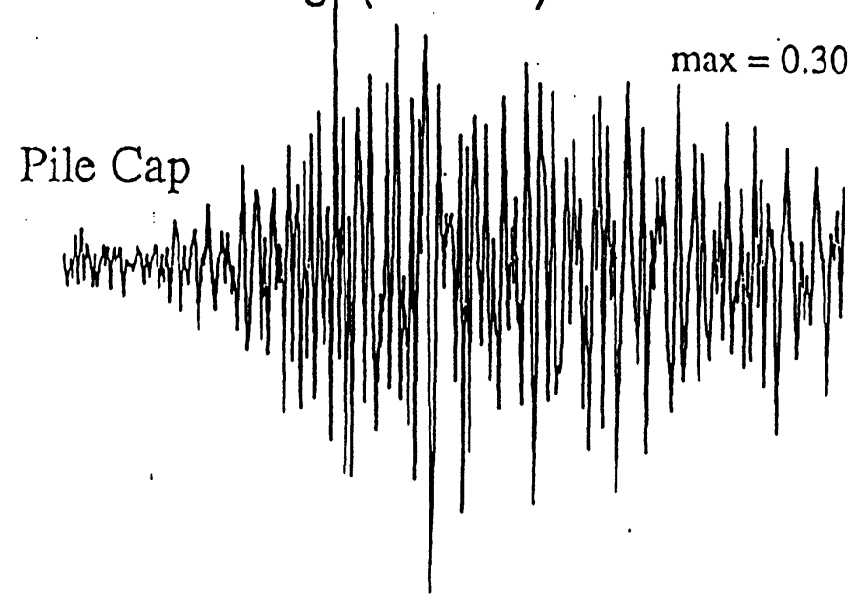

Figure 22: Recordings of acceleration from the top to the bottom of the borehole at pier 1 and from the top of the pile cap at pier 27 along the Dumbarton Bridge. 